\title{
Optical characterization of compound semiconductors using photoconductivity and photoreflectance
}

\author{
Vladimir Alexandru Stoica \\ West Virginia University
}

Follow this and additional works at: https://researchrepository.wvu.edu/etd

\author{
Recommended Citation \\ Stoica, Vladimir Alexandru, "Optical characterization of compound semiconductors using \\ photoconductivity and photoreflectance" (2000). Graduate Theses, Dissertations, and Problem Reports. \\ 1074. \\ https://researchrepository.wvu.edu/etd/1074
}

This Thesis is protected by copyright and/or related rights. It has been brought to you by the The Research Repository @ WVU with permission from the rights-holder(s). You are free to use this Thesis in any way that is permitted by the copyright and related rights legislation that applies to your use. For other uses you must obtain permission from the rights-holder(s) directly, unless additional rights are indicated by a Creative Commons license in the record and/ or on the work itself. This Thesis has been accepted for inclusion in WVU Graduate Theses, Dissertations, and Problem Reports collection by an authorized administrator of The Research Repository @ WVU. For more information, please contact researchrepository@mail.wvu.edu. 


\title{
Optical Characterization of Compound Semiconductors Using Photoconductivity and Photoreflectance
}

\author{
Vladimir A. Stoica \\ Thesis submitted to Eberly College of Arts and Sciences \\ at West Virginia University \\ in partial fulfillment of the requirements \\ for the degree of \\ Master of Science \\ in \\ Physics
}

Thomas H. Myers, Ph.D. Chair

Larry E. Halliburton, Ph.D.

David Lederman, Ph.D.

Department of Physics

Morgantown, West Virginia

2000

Keywords: Photoreflectance, Photoconductivity, GaN, CdZnTe, Mapping 


\section{ABSTRACT \\ Optical Characterization of Compound Semiconductors Using Photoconductivity and Photoreflectance}

\section{Vladimir A. Stoica}

Custom photoreflectance modulation spectroscopy and photoconductivity spectroscopy set-ups were constructed and used to characterize semiconductor materials. The ternary $\operatorname{Cd}_{1-\mathrm{x}} \mathrm{Zn}_{\mathrm{x}} \mathrm{Te}$ compound was studied to achieve fine control over its composition to provide lattice-matched substrates for the growth of $\mathrm{Hg}_{1-\mathrm{x}} \mathrm{Cd}_{\mathrm{x}} \mathrm{Te}$ which is used in infrared detectors. Photoreflectance spectroscopy, capable of accurate estimations of energy levels in semiconductors, was applied to determine composition in CdZnTe standards via band gap energy determinations. The excitonic contributions at room temperature were shown to be in agreement with literature data. The lack of lattice matched substrates for the growth of $\mathrm{GaN}$ is still providing material with considerable defect densities despite substantial efforts in material research to date. Photoconductivity spectroscopy was used to characterize various epitaxially grown GaN samples via studying defects and imperfections present in the material. Distinct photoconductance features were observed near the GaN band edge, being attributed to sub-band imperfection levels. 


\section{Acknowledgments}

I am truly indebted to my wife and life partner, Dana, for her uninterrupted love, patience and encouragement that made possible my whole dedication toward the present study.

My deepest appreciation and gratitude is dedicated to my research advisor, Dr. Thomas H. Myers. I recognize that he is a preeminent professor and specialist as well as being a good person, providing me continuous support and guidance along the whole way of this work. He has set a positive example that is one I haven't encountered many times in my life and one I will never forget.

I would like to thank Dr. Nancy Giles and Dr. Larry Halliburton for providing me assistance through the use of their lab equipment or data taken in their labs. I would also like to thank Luke Holbert and Dr. Monica Moldovan for doing experiments and providing me data that represent important complementary information to the methods I used.

Special thanks go out to Aaron Ptak not only for growth and preparation of the samples but also for useful discussions related to the common research area.

I would like to acknowledge Dr. Larry Halliburton and Dr. David Lederman for taking the time to serve on my committee. Especially, I want to thank to Dr. David Lederman for spending a lot of time with me discussing research-related matters.

Finally, I thank my family and my colleagues and teachers around the department that helped and supported me during my study.

The work performed on GaN was supported by the Office of Naval Research on Grant N00014-96-1-1008. The work performed on CdZnTe was part of a National Institute of Advanced Technology Program, "Chemical Imaging for Semiconductor Metrology", in collaboration with eV-Products, Inc. and ChemIcon, Inc. 


\section{Table of Contents}

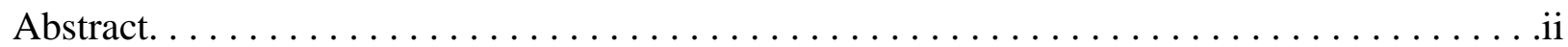

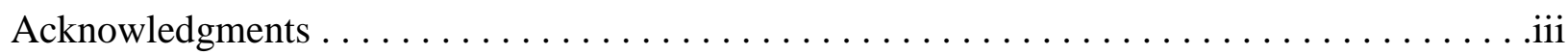

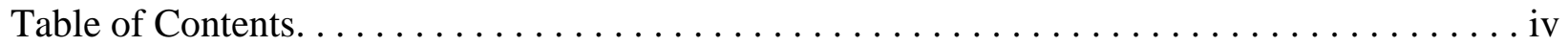

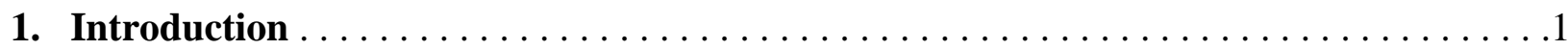

1.1 Impact of Semiconductor Research $\ldots \ldots \ldots \ldots \ldots \ldots \ldots \ldots \ldots \ldots \ldots \ldots \ldots \ldots \ldots \ldots \ldots$

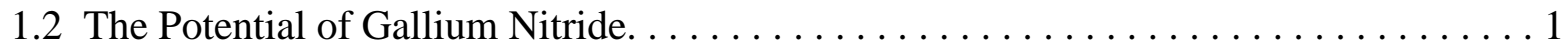

1.3 CdTe and CdZnTe Materials for Detectors $\ldots \ldots \ldots \ldots \ldots \ldots \ldots \ldots \ldots \ldots \ldots \ldots \ldots \ldots \ldots$

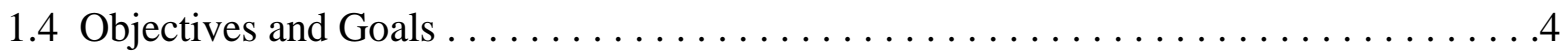

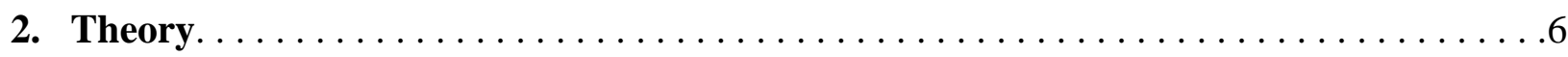

2.1 Photoreflectance Modulation Spectroscopy $\ldots \ldots \ldots \ldots \ldots \ldots \ldots \ldots \ldots \ldots \ldots \ldots \ldots \ldots$

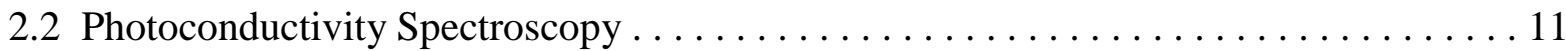

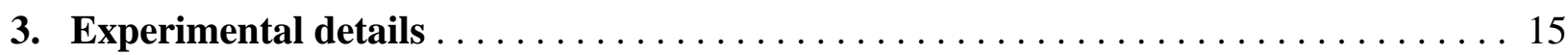

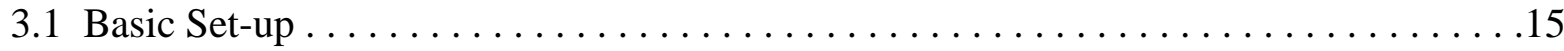

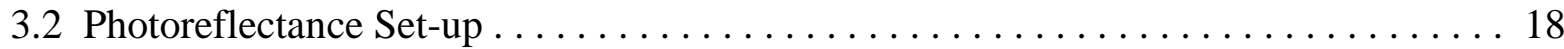

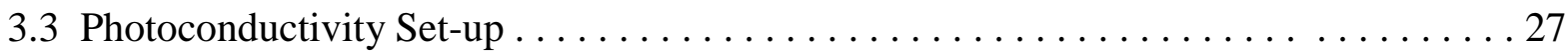

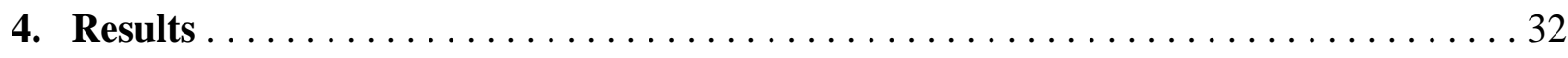

4.1 Concentration determination of small amounts of $\mathrm{Zn}$ in $\mathrm{Cd}_{1-\mathrm{x}} \mathrm{Zn}_{\mathrm{x}}$ Te bulk semiconductors

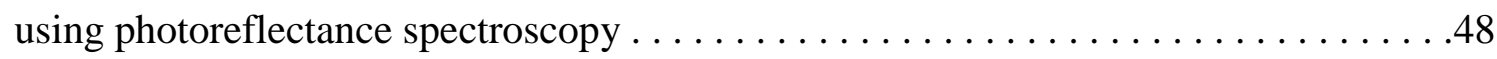

4.2 Photoconductivity spectroscopy studies of epitaxially grown $\mathrm{GaN}$ using various growth

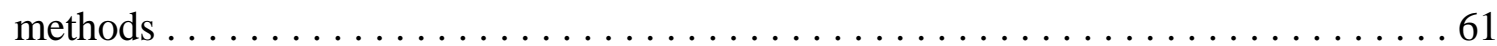

5. Summary and suggested future research $\ldots \ldots \ldots \ldots \ldots \ldots \ldots \ldots \ldots \ldots \ldots \ldots \ldots \ldots$

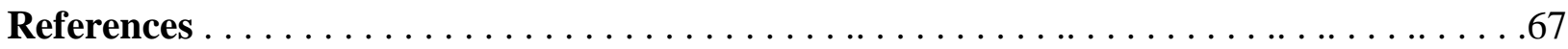




\section{INTRODUCTION}

\subsection{Impact of Semiconductor Research}

In the last century considerable advances have been realized in the research and applications of semiconductors. It is well known that semiconductor technology has a great impact on our society. We know the power of television or the personal computers that are in almost everyone's house or office, changing the way we communicate. Semiconductor applications are present everywhere around us: on roads, in houses, on your desk, even in your pocket. When a new material or semiconductor device is produced, it often opens never before imagined gates or connections that influence your life. This immediate application is why semiconductor research attracts more and more interest at all scientific levels.

\subsection{The Potential of Gallium Nitride}

More than a promising material for optoelectronics and electronics, GaN already represents an economic reality. Widely explored in the last ten years, with a publication rate that increased by more than $200 \%$ each year, $\mathrm{GaN}$ development has gone from an exploratory research activity to producing an industrial product. Tremendous effort has been carried out to understand the physical properties of nitrides so that this has been by far the most active semiconductor research field during the latter part of this decade. ${ }^{1}$

Due to promising properties such as excellent conductivity, large breakdown field, and resistance to chemical attack, GaN represents an ideal candidate for electronic devices capable of operation at high power levels, high temperatures and in caustic environments. The need to tolerate high temperature and hostile environments required by industry application including aerospace, automotive, petroleum and others has stimulated many researchers to turn their attention to $\mathrm{GaN}$ as a material candidate to meet these requirements. ${ }^{2} \mathrm{GaN}$ transistors have already been demonstrated ${ }^{3}$ that work at temperatures up to $600{ }^{\circ} \mathrm{C}$.

Increased attention toward the wide band gap semiconductors such as GaN is also due in part to the desire to create electro-optical devices operating at blue and ultraviolet (UV) wavelengths. Development of semiconductor device technology for applications in the blue 
region of the electromagnetic spectrum would allow production of the three primary colors of the visible spectrum that would have a major impact on graphic and imaging applications.

Many companies are working on nitride lasers for optical storage, since their short wavelength produces a smaller spot size when the beam is focused by an optical system. This smaller laser spot can read and write features which are closer together, allowing more data marks to be put into the disk. Since the diffraction-limited spot size is proportional to the laser wavelength, which in case of GaN is about $360 \mathrm{~nm}$, such UV light allows a spot smaller than today's widely used lasers, which typically use infrared light (780 to $850 \mathrm{~nm}$ ) or red light (650 to $670 \mathrm{~nm}) .{ }^{4}$ The recently produced GaN-based blue lasers operating at a shorter wavelength (460 to $410 \mathrm{~nm}$ ) already have the capability to overcome performance limitations of the classical red lasers used for the optical storage of information. In addition, the shorter wavelength of the blue laser can also be used in laser printers to increase the resolution. The predicted increase in the amount of information stored using blue lasers is by a factor of 20 for the case of CDs and by a factor of 6 in case of DVDs. Although blue lasers based on solid state technology have demonstrated the advantage of blue or UV light, they are not light and compact enough for consumer electronics. Research is ongoing to produce semiconductor laser diodes (LDs) and light emitting diodes (LEDs) that offer a better cost/performance/size solution. ${ }^{5}$ The most successful material used to produce green and blue LEDs is InGaN, completely dominating the market. White and yellow LEDs using GaN are also being commercialized. These products, with high light emission efficiency, should find wide use in applications such as full color displays, traffic signs and automobile and aircraft lighting. ${ }^{6}$ Violet LDs with continuous wave operation have also been recently demonstrated, having a lasing output power of $5 \mathrm{~mW}{ }^{7}$

Wide band gap compounds using GaN have important applications in communications. By creating lasing and detection devices that operate in the $240-280 \mathrm{~nm}$ range, the earth's atmosphere could be used as an effective communications screen. This is due to the large degree of absorption of the ozone layer in this wavelength range that renders the atmosphere nearly opaque to these devices. This would allow space to space communications to be secure from the earth and also allow imaging array detectors to provide sensitive surveillance of objects coming up through the atmosphere. ${ }^{8}$

Also of great importance are $\mathrm{GaN}$ photoconductive devices that are highly suited as solar-blind UV photodetectors for applications such as missile detection, flame sensing and solar 
UV monitoring. GaN-based materials are ideal for these applications due to their wide and direct band gaps, making detectors transparent to visible and infrared radiation. ${ }^{9,10}$ For the case of military application such as missile launching detection, tracking and interception, the detectivity has to be extremely large, and the spectral selectivity (how well it rejects solar light) must be as high as possible. This means that the spectral response of the device should have a flat responsivity with a sharp band edge transition and no discernable responsivity below the band gap, which is an important property for solar-blind detectors. In civilian applications, flame detectors and sensors are needed for monitoring the combustion in large industrial burners and boilers. In order to observe the flame in a daylight environment, solar blind detectors are needed. In the deeper ultraviolet ( 225 to $275 \mathrm{~nm}$ ), pilot landing aid systems have been proposed based on UV lights put on the edges of runways and taxiways, with a UV camera on board. Since this wavelength is much smaller than rain or fog particles, the light can propagate over reasonable distances. Initial evaluation has shown that visibility can gain a factor of three when going from visible to the ultraviolet. Another application for UV detectors is the dosimeter for personal use in UV-rich environments such as beaches or mountains.

Looking at the main applications produced to the date, the field of $\mathrm{GaN}$ has been and will remain in the near future driven by optical sources and detectors in the violet, blue and green, where other choices of materials have failed to provide similar performance. The market for $\mathrm{GaN}$ based optoelectronic products exists with the potential for large production volumes. At the same time, other applications are still under development and will take advantage of the large progress made in the area of optoelectronics. ${ }^{1}$

\subsection{CdTe and CdZnTe Materials for Detectors}

Considerable progress has been made in II-VI semiconductor material and in methods for improving performance of the associated radiation detectors. New high-resistivity CdZnTe (CZT) material, contact technologies, detector structures, and electronic correction methods have opened the field of nuclear and x-ray imaging for industrial and medical applications. The physical properties of $\mathrm{CdTe}$ (CT), including its unique combination of high atomic number providing good photon quantum efficiency, wide band gap and high resistivity leading to low leakage currents, good lifetime-mobility product and room temperature operation, make it an 
attractive material for $\mathrm{x}$-ray and $\gamma$-ray detectors. Keeping in mind the quality and properties of the crystals required by the applications, advances were made in the growth of bulk CdTe or $\mathrm{Cd}_{1-\mathrm{x}} \mathrm{Zn}_{\mathrm{x}} \mathrm{Te}$ using new methods such as the one recently developed by Digirad, Inc. and eVProducts, Inc. based on the high pressure Bridgeman method (HPBM). ${ }^{11}$

With recent success made by the crystal growers, a large variety of detection applications using CT and CZT materials are possible at the present in the fields of medicine, industry, astronomy and security. ${ }^{11-14}$ Spectrometers using $\mathrm{CT}$ and CZT are considered as a replacement to scintillation detectors, since they posses a higher energy resolution. Although CT and CZT detectors have been already demonstrated, continuing effort is still underway to improve the characteristics of both CT and CZT materials in order to achieve reproducible detectors.

Beside the detection application of CZT materials, this ternary semiconductor alloy also has promising applications in a variety of solid state devices such as solar cells and light emitting diodes because its band gap is tunable from 1.5 to $2.3 \mathrm{eV}$ by controlling the stoichiometry. Moreover, at the present, $\mathrm{Cd}_{1-\mathrm{x}} \mathrm{Zn}_{\mathrm{x}} \mathrm{Te}$ substrates are the favored material for epitaxial deposition of the important infrared detector material $\mathrm{Hg}_{1-\mathrm{x}} \mathrm{Cd}_{\mathrm{x}} \mathrm{Te}$ (MCT). Its popularity relies on the possibility of lattice matching and has been found that using $\mathrm{Cd}_{1-\mathrm{x}} \mathrm{Zn}_{\mathrm{x}} \mathrm{Te}$ with $\mathrm{x} \approx 0.04$ optimizes lattice matching between these two crystals. In this situation, improvements in the material quality can be obtained by reducing the density of defects associated with misfit dislocations in the MCT layer. ${ }^{15}$ This critical need to produce high quality CZT material and its later use as a substrate for epitaxial growth results in the need for contactless and nondestructive characterization techniques. The proposed techniques should be capable of revealing the required structural or optical properties for the applications mentioned above, and at the same time to provide fine control over the composition, which is necessary to achieve such applications.

\subsection{Objectives and Goals}

Automation of experimental systems capable of collecting large amounts of data is an efficient approach helping the researcher to make rapid progress toward a specific goal. Not only the continuously increasing number of samples produced by molecular beam epitaxy (MBE) growers from West Virginia University but also the number of samples coming from other labs 
has introduced the necessity to quickly investigate individual sample characteristics. This helps optimize the growth processes in order to obtain high quality materials.

Optical characterization methods for analyzing semiconducting materials have been successfully used in the past such as spectral photoconductivity ${ }^{16}$ (SPC) and modulation photoreflectance $^{17}$ (PR) techniques. These two methods proved to be an effective method of determining the sample quality and revealing the role of imperfections or impurities, which is in agreement with West Virginia University MBE Lab characterization necessities. At the same time the PR technique is a tool capable of making accurate determination of the band gap energies of compound semiconductors which are directly correlated with the relative composition.

Accordingly, the following goals were determined, which motivated the research presented in this thesis:

1. To construct a custom PR set-up capable of accurately determining energy levels in semiconductors.

2. To construct a custom SPC setup to study optical properties of GaN to reveal impurity and defect states in the band gap and to correlate these features with other optical characterization techniques.

3. To characterize a set of CdZnTe standards by determining and mapping the sample composition across the surface, using the capability for accurate band gap determination of the PR technique 


\section{THEORY}

\subsection{Photoreflectance Modulation Spectroscopy}

The photoreflectance technique is a modulation spectroscopy based on very general principles of experimental physics. Instead of directly measuring the optical spectrum (reflectance or transmittance), the derivative with respect to some parameter is evaluated. This can be accomplished by modulating some property of the sample or measuring a system in a periodic fashion and measuring the corresponding change in the reflectance. Examples of the perturbations, which correspond to different modulation methods, are changes in the electric or magnetic field, heat pulse, or uniaxial stress. Such repetitive perturbations modify the spectral response of the sample, giving rise to differential-like spectra in the region where optical excitation processes occur. Because of this derivative-like nature, a large number of sharp spectral features can be observed in modulated reflectance spectra of semiconductors even at room temperature. With these sharp, well-resolved spectra, it is possible to analyze and directly yield the properties of the material under study. Changes in reflectance as small as $10^{-6}-10^{-7}$ can be observed using phase -sensitive techniques. The ability to perform a lineshape fit is one of the great advantages of photoreflectance modulation spectroscopy. Since, for the modulated signal, the features are localized in photon energy, it is possible to account for the lineshapes to yield accurate values of important parameters such as energies and broadening functions of interband (intersubband) transitions. For example, even at $300 \mathrm{~K}$ it is possible to obtain the energy of a particular feature to within a few meV. The extensive fundamental experimental and theoretical work in the area of modulation spectroscopy during the last 25 years, particularly the electroreflectance and photoreflectance methods, have provided the necessary framework to develop the technique into a powerful tool for material, device and processing characterization. ${ }^{17-}$ ${ }^{23}$ In this thesis, the application of photoreflectance spectroscopy to determine topographical variations in composition of compound semiconductors is considered.

In the photoreflectance technique, a modulated laser beam with an energy above the band gap of the material being studied creates the photoinduced variation in the built-in electric field. The experimental details are considered in the next chapter. The field modulation mechanism ${ }^{23}$ is depicted in Figure 2.1, for a case of an n-type semiconductor. Because of the pinning of the 


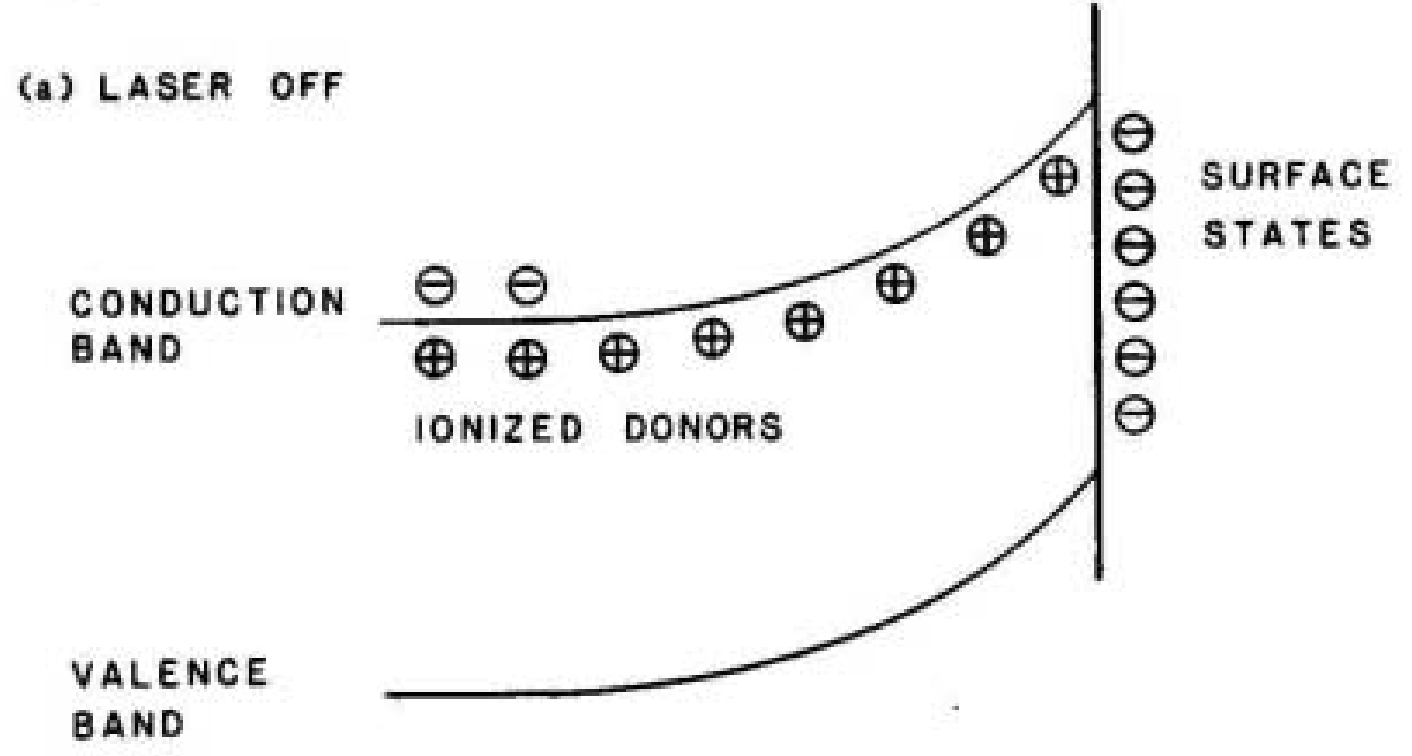

(b) LASER ON

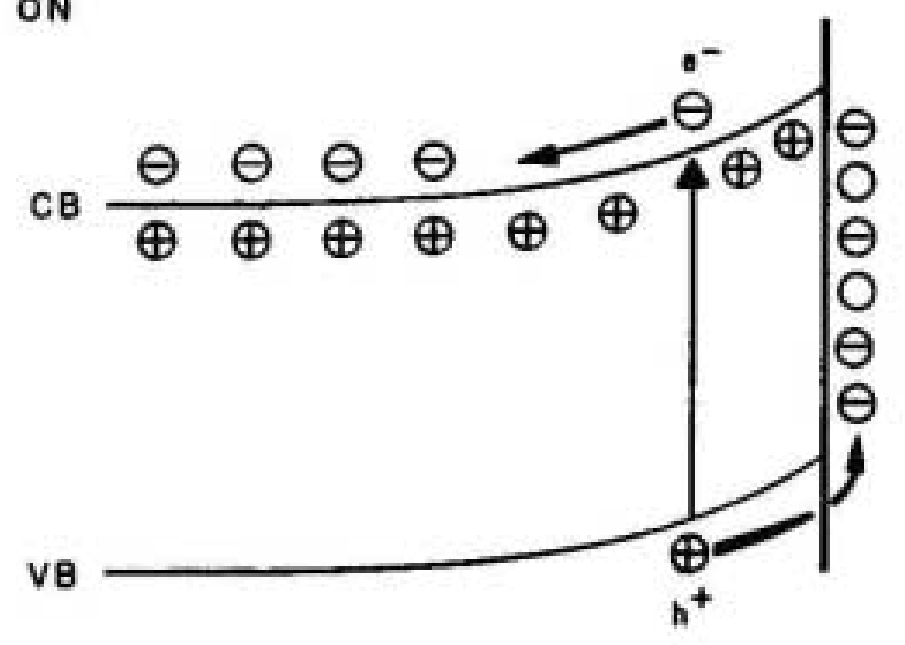

Figure 2.1 Schematic representation of the photoreflectance effect for an n-type sample. In a) the sample is in the dark, and the Fermi-level pinning to surface states induces a built-in field. In b) photoexcited carriers separate in the field, with minority carriers neutralizing charge in surface states (after Reference 23). 
Fermi energy at the surface, there exists a space-charge layer. The occupied surface states contain electrons from the bulk. Photoexcited electron-hole pairs are separated by the built-in field, with the minority carrier (holes for this case) being swept toward the surface. At the surface, the holes neutralize the trapped charge, reducing the built-in field. The surface field modulation is resolved in the reflection spectra and it has been shown to be sensitive to critical point transitions in the Brillouin zone, with the resulting spectrum exhibiting sharp derivativelike features and typically a featureless background. Also, weak features that may have been difficult to observe in the absolute reflection or absorption spectrum can be enhanced. While it is difficult to calculate a full reflectance spectrum, this is not the case for modulation spectroscopy. For well-known critical points in the Brillouin zone, it is possible to account for the lineshapes in a modulation spectrum. ${ }^{17}$

Differential changes in the reflectivity can be related to the perturbation of the complex dielectric function $\left[\varepsilon\left(=\varepsilon_{1}+i \varepsilon_{2}\right)\right]$ through the following relationship: ${ }^{17-23}$

$$
\Delta \mathrm{R} / \mathrm{R}=\mathrm{a}\left(\varepsilon_{1}, \varepsilon_{2}\right) \Delta \varepsilon_{1}+\mathrm{b}\left(\varepsilon_{1}, \varepsilon_{2}\right) \Delta \varepsilon_{2}
$$

where $\mathrm{a}$ and $\mathrm{b}$ are the Seraphin ${ }^{19}$ coefficients, related to the unperturbed dielectric function, and $\Delta \varepsilon_{1}$ and $\Delta \varepsilon_{2}$ are the changes in the complex dielectric function due to the perturbation. The Seraphin coefficients a and $\mathrm{b}$ can be written as: ${ }^{20}$

$$
a=\frac{1}{R} \frac{\partial R}{\partial \varepsilon_{1}} ; \quad b=\frac{1}{R} \frac{\partial R}{\partial \varepsilon_{2}}
$$

Near the fundamental gap of bulk material, $\mathrm{b} \approx 0$, so that $\Delta \mathrm{R} / \mathrm{R} \approx \mathrm{a} \Delta \varepsilon_{1}$ is the only significant term. The quantities $\Delta \varepsilon_{1}$ and $\Delta \varepsilon_{2}$ are related by Kramers-Kronig inversion. The functional form of $\Delta \varepsilon_{1}$ and $\Delta \varepsilon_{2}$ can be calculated for a given perturbation provided that the dielectric function and critical point are known.

Photoreflectance spectroscopy follows the theoretical treatment applied for the electromodulation that can be classified into two categories, i.e. high- and low-field regimes, depending on the relative strength of certain characteristic energies. ${ }^{20}$

In the high-field case $|\hbar \theta| \geq \Gamma$, the band structure is unchanged and the spectra lineshape contains Franz-Keldysh oscillations, ${ }^{24}$ where $\Gamma$ is the broadening parameter and $\hbar \theta$ is the electro-optic energy given by:

$$
(\hbar \theta)^{3}=q^{2} \hbar^{2} F^{2} / 2 \mu_{\amalg}
$$


where $\mathrm{F}$ is the magnitude of the electric field and $\mu_{\mathrm{II}}$ is the reduced interband effective mass in the direction of the field. This high field regime is not appropriate for studying near band gap transitions and thus will not be considered in detail in the present study.

The low-field case is defined by $|\hbar \theta| \leq \Gamma$, and this regime is probed by our PR experiment case. It has been demonstrated for this condition that the third-derivative modulation is an adequate method to describe the behavior of the involved phenomena in electromodulation spectroscopy. ${ }^{25}$ In this case the band-to-band transitions in the bulk material are involved and electromodulation can destroy the translational symmetry of the material, being able to accelerate unbound electron and/or holes. The time-dependent Schrodinger equation for an electron in the presence of a uniform electric field F can be written as: $:^{20,25}$

$$
\left(H_{0}+e \vec{F} \cdot \vec{r}\right) \psi=i \hbar \frac{\partial \psi}{\partial t}
$$

where the crystal Hamiltonian $\mathrm{H}_{0}$, with crystal potential $\mathrm{V}(\mathrm{r})$, is:

$$
H_{0}=\frac{p^{2}}{2 m_{0}}+V(r)
$$

The time evolution of the wavefunction, which at time $\mathrm{t}=0$ is the Bloch function $\Psi_{\mathrm{n}}\left(\mathrm{k}_{0}, \mathrm{r}\right)$, is given by:

$$
\psi_{n}(k, r, t)=\exp \left(-i \frac{\left(H_{0}+e \vec{F} \cdot \vec{r}\right) t}{\hbar}\right) \psi_{n}\left(k_{0}, r\right)
$$

The main effect of the electric field is to change the wavevector from $\vec{k}_{0}$ to $\vec{k}_{0}-e \vec{F} t / \hbar$. As a result, states with $\vec{k}$ vector along the field direction are mixed. The solution of the time dependent Schrodinger equation can be approximately written as:

$$
\psi_{n}(k, r)=\exp \left(-\frac{i}{\hbar} \int E_{n}(k) d t\right) \psi_{n}(k, r, t)
$$

where $\vec{k}(t)=\vec{k}_{0}-e \vec{F} t / \hbar$.

It is possible to determine the optical constants in the presence of an electric field by taking into account the time dependence of $\vec{k}$ and the broadening parameter $\Gamma$. Considering the case of a weak electric field, F, or a large broadening $\Gamma$ parameter (in both cases having $\Gamma>$ $\hbar \theta$ ), the real and imaginary parts of the dielectric function are obtained: ${ }^{20}$ 


$$
\varepsilon_{i}(E, F, \Gamma)=\varepsilon_{i}(E, 0, \Gamma)+\frac{(\hbar \theta)^{3}}{12} E^{2} \frac{\partial^{3}}{\partial E^{3}}\left[E^{2} \varepsilon_{i}(E, 0, \Gamma)\right]+\ldots
$$

where $\mathrm{i}=1,2$ gives the indices for real or imaginary part of the dielectric function.

It can be concluded that the low-field electromodulation $(|\hbar \theta|<<\Gamma)$ gives a third derivative spectroscopy for band-to-band transitions and the change in the complex dielectric function is correspondingly given by:

$$
\Delta \varepsilon=\frac{(\hbar \theta)^{3}}{12 E^{2}} \frac{\partial^{3}}{\partial E^{3}}\left[E^{2} \varepsilon(E, 0, \Gamma)\right]
$$

In the case when the distribution of states near the critical point exhibits a Lorentzian type dispersion, then $\varepsilon(E, 0, \Gamma)$ will have a Lorentzian shape and $\Delta \varepsilon$ in Equation 2.10 can be computed. Ultimately, Equations 2.1 and 2.10 can be written in the simplified form when this is true:

$$
\left.\Delta R / R=\operatorname{Re} \mid C e^{i \phi}\left(E-E_{g}+i \Gamma\right)^{-m}\right\rfloor
$$

where $\mathrm{C}$ is the amplitude, $\phi$ is a phase angle that accounts for the mixture of real and imaginary components of $\varepsilon$ in Equation 2.1 as well as the influence of non-uniform electric fields, interference and electron-hole interaction effects, and $E_{g}$ is the band gap energy. The parameter $\mathrm{m}$ in Equation 2.11 depends on the type of critical point. For three-dimensional critical points, such as the direct gap of CdTe or $\mathrm{Cd}_{1-\mathrm{x}} \mathrm{Zn}_{\mathrm{x}}$ Te materials that are studied in this thesis, $\mathrm{m}=2.5$.

However, if bound states such as excitons in the bulk are to be considered, then the perturbing electric field does not accelerate electrons and/or holes. These particles do not have translational symmetry and are confined in space. Their energy spectrum is discrete and the modulating field can alter the binding energy of the particle. ${ }^{17}$ For confined systems, the energies are discrete and result in an infinite effective mass in that direction. An applied electric field adds a linear potential, which tilts the confining potential, changing the shape. The electrons and holes become spatially polarized, but still remain confined. This alters both the electronic energies and the wavefunction overlap (intensity, I). Also the tilting of the potential can result in a change in lifetime as a result of tunneling that modifies $\Gamma$.

In terms of electromodulation, the infinite mass means that Equation 2.10 is no longer applicable, since $\hbar \theta=0$. Under these conditions the change in the dielectric function induced by the modulation field, $\mathrm{F}_{\text {a.c. }}$, is first-derivative and not the third derivative as for 3D systems. For 
this case, it has been shown that $\Delta \varepsilon$ can be expressed in a compact manner as a first derivative functional form for the modulated dielectric function: ${ }^{17,20}$

$$
\Delta \varepsilon=\left[\frac{\partial \varepsilon}{\partial E_{g}} \frac{\partial E_{g}}{\partial F_{a . c .}}+\frac{\partial \varepsilon}{\partial \Gamma} \frac{\partial \Gamma}{\partial F_{a . c .}}+\frac{\partial \varepsilon}{\partial I} \frac{\partial I}{\partial F_{\text {a.c. }}}\right] F_{\text {a.c. }}
$$

where $\partial E_{g} / F_{\text {a.c. }}$ is the change in energy due to the Stark effect, $\partial \Gamma / F_{\text {a.c. }}$ is the change in broadening parameter due to a variation of the lifetime, and $\partial I / F_{a . c .}$ is the change in intensity due to redistribution of charges.

Again, if the distribution of states near the critical point exhibits a Lorentzian type dispersion and the third term of Equation 2.12 can be neglected, combining Equation 2.1 with Equation 2.12 can be written as: ${ }^{17,20}$

$$
\Delta R / R=\operatorname{Re}\left[C e^{i \phi}\left(E-E_{g}+i \Gamma\right)^{-2}\right\rfloor
$$

for the case of excitonic transitions. In the case of a Gaussian dispersion near the critical point, the expression for the lineshape is more complicated in relation to Equation 2.13 and is given in Reference 17.

In the present work only the Lorentzian line profiles will be considered in Chapter 4 since Equations 2.11 and 2.13 are good descriptions for the near band gap transitions that are measured at room temperature with photoreflectance spectroscopy.

\subsection{Photoconductivity Spectroscopy}

Photoconductivity (PC) is an important property of semiconductors by means of which the conductivity of the sample changes due to incident radiation. The spectroscopy based on PC represents an alternative to regular absorption spectroscopy. Photoconductivity is an elementary process in solids, and as the name suggests, it involves the generation and recombination of charge carriers and their transport to the electrodes. The phenomenon of PC also includes the thermal carrier relaxation process, charge carrier statistics, effects of electrodes, and several mechanisms of recombination. As every mechanism mentioned here is complicated, PC in general is a very complex process. For interpretation purposes, complementary information from other experimental techniques such as photoluminescence, optical absorption, and electroreflectance is often needed. Despite the complexity of the photoconductance process, PC 
spectroscopy is able to provide useful and valuable information about physical properties of materials and offers applications in photodetection and radiation measurements. ${ }^{26}$

Taking place by photon absorption into the material, the photoconductivity process occurs as a result of several mechanisms that compete with generation of the PC carriers. Bandto-band transitions, impurity levels to band edge transitions, ionization of donors, and deep-level (located in the valence band) to conduction band transitions are possible phenomena which may contribute or dominate the PC signal with respect to the energy range and the material band structure characteristics. When the energy of the incident photons on material is greater than the band gap, it will create electrons and holes in the conduction and valence bands respectively, providing the main contribution to the PC signal. If we measure PC in a doped semiconductor and the photon energy is slightly less than the band gap then the impurity atom can absorb the photon, creating a free electron in the conduction band. In this case photoresponse starts from the low-energy side of the band gap and photoconduction occurs due to excitation near the band edge. It is also possible to observe photoconductivity when the energy of the incident photon is much smaller than that of the band gap. When the energy of the incident photons matches the ionization energy of the impurity atoms, they are ionized, creating extra electrons in the conduction band, and hence an increase in the conductivity is observed.

Whatever the main contribution to the PC signal is at a specific energy, PC is due to the absorption of photons, either by an intrinsic process or by impurities with or without phonons, leading to the creation of free charge carriers in the conduction band and/or the valence band.

The measurement of the photoconductivity effect requires the application of electrical contacts and an electric field to measure the photogenerated carriers in the material under investigation at the contacts (see the experimental set-up described in the Section 3.3). In this configuration, the photoconductivity is related to the change in the dark electrical conductivity, which is described by:

$$
\sigma=e\left(n \mu_{n}+p \mu_{h}\right)
$$

where $e$ is the electron charge, $n$ and $p$ are the density (number per unit volume) of free electrons and holes, respectively, and $\mu_{\mathrm{n}}$ and $\mu_{\mathrm{p}}$ are the electron mobility and hole mobility, respectively. If considering a homogenous material in which $\mathrm{n}, \mathrm{p}, \mu_{\mathrm{n}}$ and $\mu_{\mathrm{p}}$ are uniform throughout the material, photoconductivity results when absorbed radiation changes the values of the mentioned parameters: 


$$
\Delta \sigma=e\left(\mu_{n} \Delta n+\mu_{h} \Delta p\right)+e\left(n \Delta \mu_{n}+p \Delta \mu_{h}\right)
$$

Mechanisms exist for both changes in carrier densities: $\Delta \mathrm{n}$ and $\Delta \mathrm{p}$, and carrier mobilities: $\Delta \mu_{\mathrm{n}}$ and $\Delta \mu_{\mathrm{p}}$. However, in actual materials the first term on the right of Equation 2.15 almost always dominates. ${ }^{27}$ The second term including the carrier mobility changes will be neglected and Equation 2.15 simplifies to:

$$
\Delta \sigma=e\left(\mu_{n} \Delta n+\mu_{h} \Delta p\right)
$$

It is convenient to express the relationship between the change in carrier densities and the excitation intensity $\mathrm{f}$ (number of electron-hole pairs per second per unit volume created in the photoconductor) in terms of electron and hole lifetimes for steady-state conditions,

$$
\Delta n=f \tau_{n}, \quad \Delta p=f \tau_{p}
$$

Then taking into account experimental concerns such as reflection and absorption, we can rewrite Equation 2.16:

$$
\Delta \sigma=f e(1-R)\left(1-e^{-\alpha x}\right)\left(\mu_{n} \tau_{n}+\mu_{h} \tau_{p}\right)
$$

which shows that the mobility and lifetime are the key parameters in PC. R is the reflection coefficient, $\alpha$ is the absorption coefficient and $\mathrm{x}$ is the penetration depth of the radiation.

Another way to describe photoconductivity is to consider the change in the current produced by incident photons: ${ }^{16}$

$$
\Delta i=e f V_{0}\left(\mu_{n} \tau_{n}+\mu_{h} \tau_{p}\right) \frac{V}{L^{2}}
$$

where $\mathrm{V}_{0}$ is the volume in which radiation penetrates, $\mathrm{V}$ is the applied voltage and $\mathrm{L}$ is the distance between electrodes. In the case of the real experiment, we should consider the reflection and absorption of radiation mechanisms, and Equation 2.19 transforms into:

$$
\Delta i=e f V_{0}(1-R)\left(1-e^{-\alpha x}\right)\left(\mu_{n} \tau_{n}+\mu_{h} \tau_{p}\right) \frac{V}{L^{2}}
$$

Looking at the Equations 2.18 and 2.20 that describe the material response under the radiation excitation we may keep in mind that the photoconductivity process will be lead by the absorption and the mobility-lifetime products to generate the signal. If spectral changes are considered, the changes in the parameters mentioned above will participate to provide the overall spectral response. Carrier lifetimes, excitation or recombination contributions to PC are considered in great detail by several authors, ${ }^{16,26,27}$ taking into account and distinguishing 
between specific energy levels of the material under study. This work is limited to only a qualitative description, in which spectral photoconductivity is seen to be dominated by band-toband transitions near the band gap edge and by dopants or imperfections in the lower energy range. 


\section{EXPERIMENTAL DETAILS}

\subsection{Basic Set-up}

The experimental set-ups were based on arrangements previously described in the literature. The set-ups were realized step-by-step, via optimizing the techniques involved in this work in order to obtain meaningful final results with respect to the theory. In addition to the experimental instrumentation a computer-based system for data acquisition and experiment control was designed. The different arrangements corresponding to specific methods or calibration needs are summarized in the next subsections.

The personal computer interface was programmed using the Lab-View (National Instruments) software and available instrument drivers supplied by various instrument merchants. The hardware was controlled using an IEEE 488 interface (National Instruments PCIGPIB) using parallel communication for most instruments. Serial communication was used with the spectrometer. Careful troubleshooting was required to facilitate proper communication in sequences of sending commands and reading data from instruments. The user interface application is illustrated in Figure 3.1, displaying the spectra window for real-time visualization. The automated instruments in our set-ups were a diffraction-grating monochromator (Instruments SA HR-320) that was equipped with a stepper motor, a lock-in amplifier (Stanford Research SR510) and a DC voltmeter (Keithley 197A). The instruments were accessed by computer as in the flow chart shown in Figure 3.2. The diagram describes the execution sequences of the Lab-View program application. Specific attention was paid to solving experiment problems via programming that include the monochromator backlash and its correction, automatic change to smaller sensitivity scale when the signal was too big, and a statistical collection of data for each step of the measurement and a graphical visualization of the spectra during the experiment. The interface program permitted control over the position of the monochromator with a precision of a fraction of an angstrom with excellent reproducibility, while at the same time acquisition and storage of the data from the lock-in amplifier and DC voltmeter were performed. 


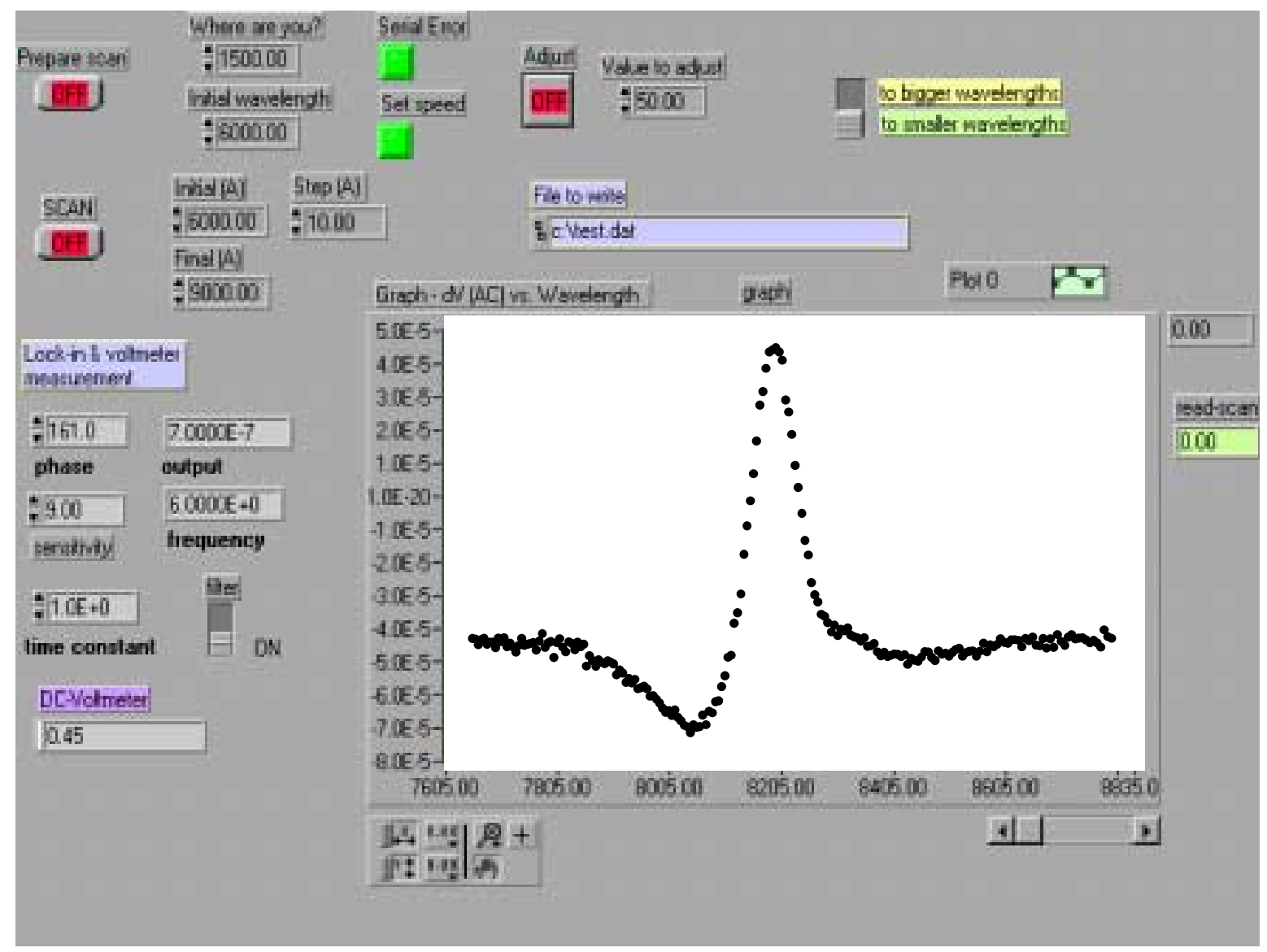

Figure 3.1 User interface of the program for data acquisition and experiment control. 


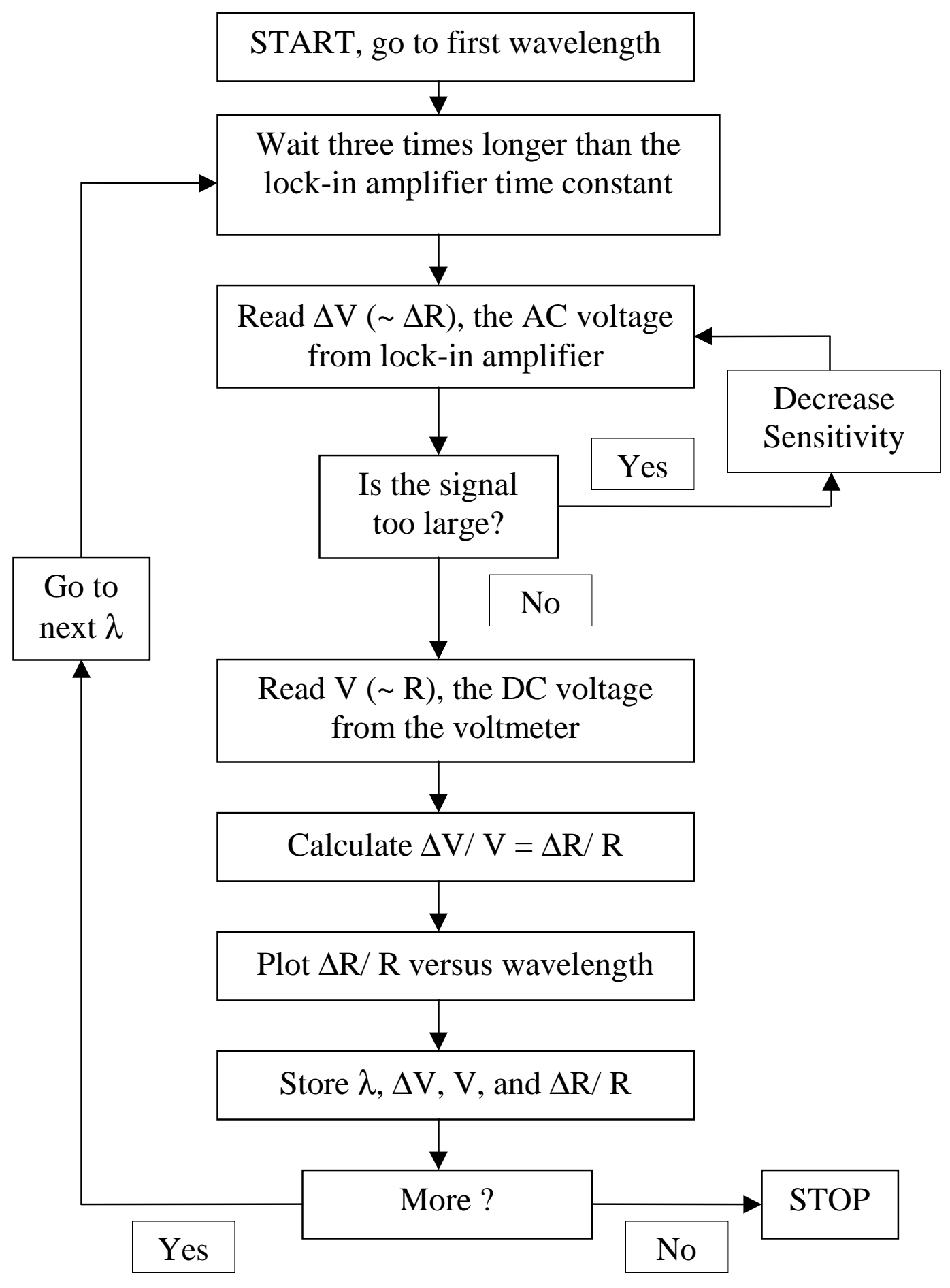

Figure 3.2 Flow chart for the data acquisition and experiment control program. 
For a good accuracy in the estimation of real wavelengths corresponding to the data, the monochromator was carefully calibrated. A mercury calibration lamp with very narrow emission lines was used as the light source at the input of monochromator to make the correspondence between the wavelength indicated by the instrument and the corresponding mercury lamp wavelengths. The light from the mercury lamp was chopped to facilitate the detection of small signals using phase sensitive detection (lock-in amplifier). The reference frequency fixed by the chopper is also used as an input for the lock-in amplifier to detect only that AC component having a frequency that is equal to the chopper reference frequency.

To observe the resolution provided by the use of a particular slit dimension, the detected peaks were fitted with Gaussian functions (Figure 3.3) to obtain the full width at half maximum (FWHM) corresponding to each slit and look at the width versus slit dependency. The differences between true wavelengths from the mercury lamp and the monochromator dial wavelengths are symbolized with $\Delta_{\lambda}$, and these values were calculated from the maximum peak positions. The change in $\Delta_{\lambda}$ versus the true wavelength is represented in Figure 3.4 and the data from the fits are found the Table 3.1. Polynomial function fits were employed to estimate the $\Delta_{\lambda}$ function variation for the entire range of considered wavelengths. The monochromator calibration furnished an accuracy of wavelength determination of about $1 \AA$.

\subsection{Photoreflectance Set-up}

We have chosen to use photoreflectance in our laboratory because it is a contactless and nondestructive technique. The method takes advantage of the application of a small periodic perturbation to a physical property of the sample. The change in optical function (reflectivity) is only a small fraction of its unperturbed value, typically 1 part in $10^{4}$ or less. The perturbation is extracted through the use of a lock-in amplifier tuned to the modulation frequency. ${ }^{28}$ The conventional photoreflectance apparatus ${ }^{17}$ uses a mechanical chopper to modulate the laser light and is represented in Figure 3.5. The modulation of the sample is produced by photoexcitation of electron-hole pairs created by the laser. The laser light will have a characteristic wavelength corresponding to radiation with an energy above the band gap of the sample studied to assure that an adequate number of electron-hole pairs are photoexcited to produce the PR signal. 


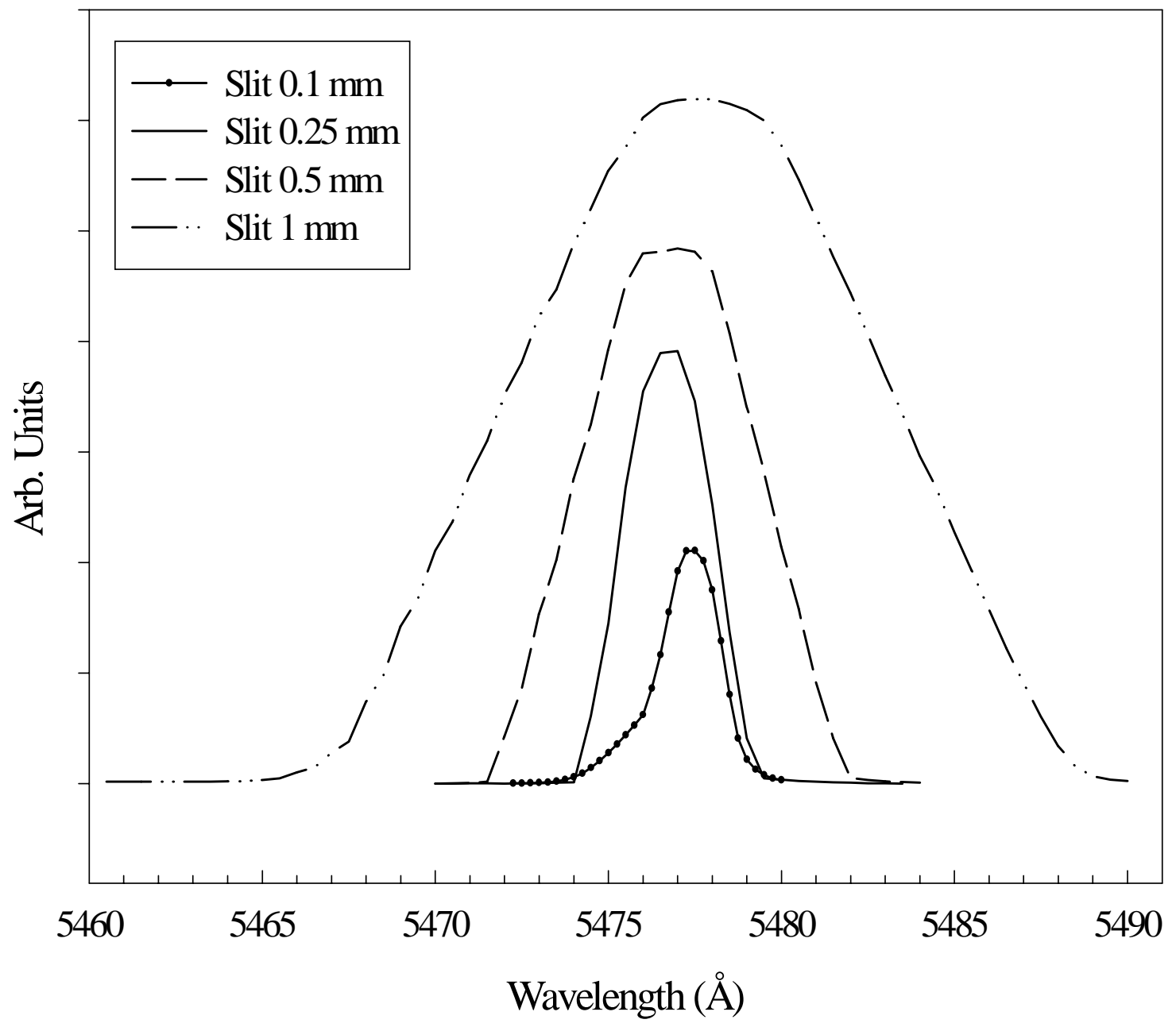

Figure 3.3 Mercury lamp line seen at the detector as a function of horizontal slit. All curves were rescaled for width comparison. 


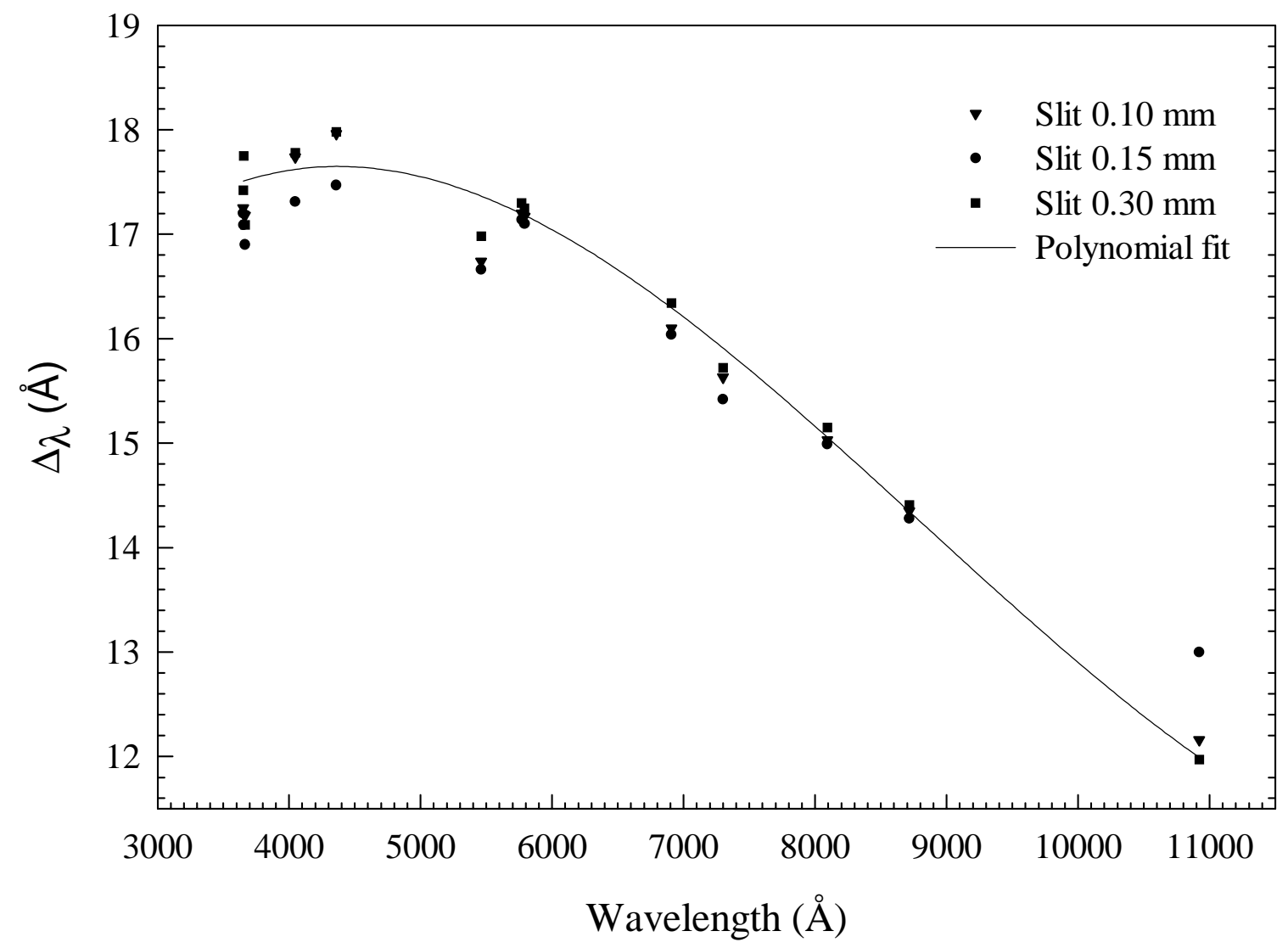

Figure 3.4 Monochromator calibration corresponding to different horizontal slits. 


\begin{tabular}{|c|c|c|c|c|}
\hline $\begin{array}{c}\text { Slit } \\
(\mathrm{mm})\end{array}$ & $\begin{array}{c}\text { Mercury lamp } \\
\text { wavelength, } \lambda_{0},(\AA)\end{array}$ & $\begin{array}{c}\text { Monochromator } \\
\text { wavelength, } \lambda_{\mathrm{e}},(\AA)\end{array}$ & $\Delta_{\lambda}=\lambda_{\mathrm{e}}-\lambda_{0},(\AA)$ & FWHM, $(\AA)$ \\
\hline \multirow[t]{13}{*}{0.5} & 3131.56 & 3147.72 & 17.74 & 5.85 \\
\hline & 3131.84 & 3148.44 & 17.46 & 4.75 \\
\hline & 3650.15 & 3665.71 & 15.56 & 5.04 \\
\hline & 3654.83 & 3668.6 & 13.77 & 5.89 \\
\hline & 3663.28 & 3679.58 & 16.3 & 8.78 \\
\hline & 4046.56 & 4063.64 & 17.08 & 6.05 \\
\hline & 4358.35 & 4375.34 & 16.99 & 4.84 \\
\hline & 5460.74 & 5476.79 & 16.05 & 4.69 \\
\hline & 5769.6 & 5786.38 & 16.78 & 4.72 \\
\hline & 5790.66 & 5807.25 & 16.59 & 4.77 \\
\hline & 6907.5 & 6923.13 & 15.63 & 4.97 \\
\hline & $4046.56 \times 2$ & 8107.3 & 14.2 & 4.54 \\
\hline & $4358.35 \times 2$ & 8730.52 & 13.82 & 4.58 \\
\hline \multirow[t]{12}{*}{0.3} & 3650.15 & 3667.57 & 17.42 & 3.85 \\
\hline & 3654.83 & 3672.58 & 17.75 & 3.36 \\
\hline & 3663.28 & 3680.37 & 17.09 & 3.91 \\
\hline & 4046.56 & 4064.34 & 17.78 & 3.68 \\
\hline & 4358.35 & 4376.33 & 17.98 & 3.74 \\
\hline & 5460.74 & 5477.72 & 16.98 & 3.65 \\
\hline & 5769.6 & 5786.9 & 17.3 & 3.67 \\
\hline & 5790.66 & 5807.91 & 17.25 & 3.74 \\
\hline & 6907.5 & 6923.84 & 16.34 & 4.3 \\
\hline & $3650.15 \times 2$ & 7316.02 & 15.72 & 3.51 \\
\hline & $4046.56 \times 2$ & 8108.25 & 15.15 & 3.5 \\
\hline & $4358.35 \times 2$ & 8731.11 & 14.41 & 3.71 \\
\hline \multirow[t]{13}{*}{0.25} & 3131.56 & 3147.66 & 16.1 & 2.54 \\
\hline & 3131.84 & 3148.2 & 16.36 & 2.75 \\
\hline & 3650.15 & 3666.76 & 16.61 & 2.45 \\
\hline & 3654.83 & 3671.56 & 16.73 & 2.3 \\
\hline & 3663.28 & 3679.73 & 16.45 & 2.79 \\
\hline & 4046.56 & 4063 & 16.44 & 2.35 \\
\hline & 4358.35 & 4375.17 & 16.82 & 2.37 \\
\hline & 5460.74 & 5476.72 & 15.98 & 2.35 \\
\hline & 5769.6 & 5786.1 & 16.5 & 2.33 \\
\hline & 5790.66 & 5807.1 & 16.44 & 2.37 \\
\hline & $3131.84 \times 2$ & 6278.73 & 15.65 & 2.37 \\
\hline & $4358.35 \times 2$ & 8730.6 & 13.9 & 2.26 \\
\hline & $5460.74 \times 2$ & 10933.45 & 11.97 & 3.05 \\
\hline
\end{tabular}




\begin{tabular}{|c|c|c|c|c|}
\hline $\begin{array}{c}\text { Slit } \\
(\mathrm{mm})\end{array}$ & $\begin{array}{c}\text { Mercury lamp } \\
\text { wavelength, } \lambda_{0},(\AA)\end{array}$ & $\begin{array}{c}\text { monochromator } \\
\text { wavelength, } \lambda_{\mathrm{e}},(\AA)\end{array}$ & $\Delta_{\lambda}=\lambda_{\mathrm{e}}-\lambda_{0},(\AA)$ & FWHM, $(\AA)$ \\
\hline $\mathbf{0 . 1 5}$ & 3650.15 & 3667.35 & 17.2 & 1.94 \\
\hline & 3654.83 & 3671.92 & 17.09 & 1.79 \\
\hline & 3663.28 & 3680.18 & 16.9 & 2.06 \\
\hline & 4046.56 & 4063.87 & 17.31 & 2.0 \\
\hline & 4358.35 & 4375.82 & 17.47 & 1.97 \\
\hline & 5460.74 & 5477.4 & 16.66 & 1.96 \\
\hline & 5769.6 & 5786.74 & 17.14 & 1.88 \\
\hline & 5790.66 & 5807.76 & 17.1 & 1.88 \\
\hline & 6907.5 & 6923.54 & 16.04 & 1.75 \\
\hline & $3650.15 \times 2$ & 7315.72 & 15.42 & 1.79 \\
\hline & $4046 \times 2$ & 8108.09 & 14.99 & 1.68 \\
\hline & $4358.35 \times 2$ & 8730.98 & 14.28 & 1.67 \\
\hline & $5460.74 \times 2$ & 10934.48 & 13 & 1.55 \\
\hline $\mathbf{0 . 1 0}$ & 3650.15 & 3667.4 & 17.25 & 1.4 \\
\hline & 3654.83 & 3672.02 & 17.19 & 1.21 \\
\hline & 3663.28 & 3680.46 & 17.18 & 1.65 \\
\hline & 4046.56 & 4064.3 & 17.74 & 1.39 \\
\hline & 4358.35 & 4376.31 & 17.96 & 1.31 \\
\hline & 5460.74 & 5477.48 & 16.74 & 1.26 \\
\hline & 5769.6 & 5786.8 & 17.2 & 1.3 \\
\hline & 5790.66 & 5807.83 & 17.17 & 1.3 \\
\hline & 6907.5 & 6923.6 & 16.1 & 1.32 \\
\hline & $3650.15 \times 2$ & 7315.93 & 15.63 & 1.1 \\
\hline & $4046 \times 2$ & 8108.13 & 15.03 & 1.1 \\
\hline & $4358.35 \times 2$ & 8731.05 & 14.35 & 1.11 \\
\hline & $5460.74 \times 2$ & 10933.64 & 12.16 & 1.55 \\
\hline
\end{tabular}

Table 3.1 Mercury lamp calibration of the monochromator. 


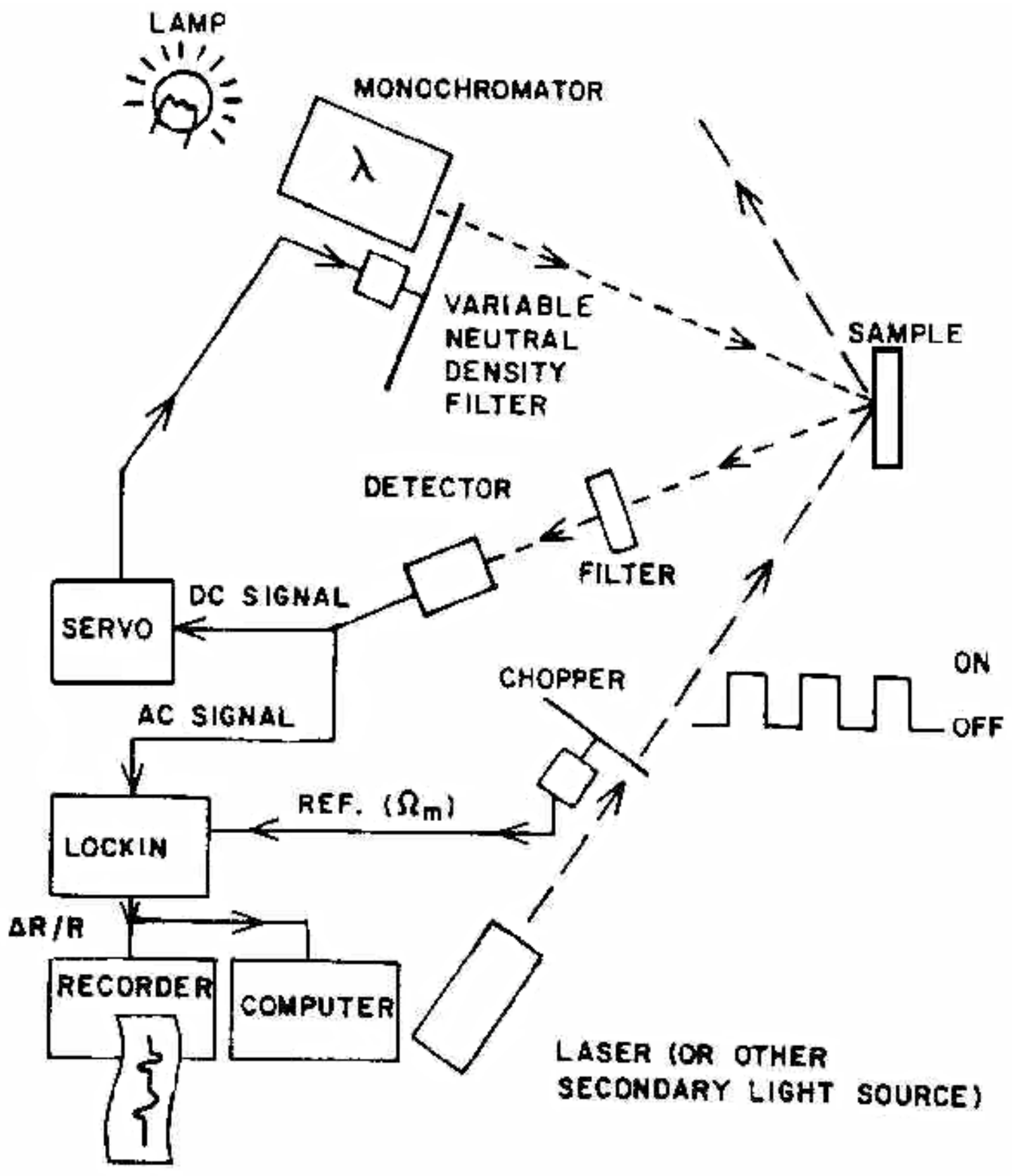

Figure 3.5 Schematic representation of a conventional photoreflectance experiment arrangement (after Reference 17). 
In addition, a monochromatic beam of light coming from the monochromator is hitting the sample surface in the same area as the impinging laser light. The detector will collect the reflected light from the sample that contains three components: a DC signal $\mathrm{I}_{0}(\lambda) \mathrm{R}(\lambda)$, an AC signal proportional with the modulated reflectance $\mathrm{I}_{0}(\lambda) \Delta \mathrm{R}(\lambda)$ and another $\mathrm{AC}$ signal $\Delta \mathrm{I}_{\mathrm{PL}}(\lambda)$ that represents the photoluminescence (PL) signal, where $R(\lambda)$ is the unperturbed reflectance of the sample and $\Delta R(\lambda)$ is the modulated reflectance. Because the PL signal entering the detector will have the same frequency as the modulated reflectance, its contribution will mix with the useful reflectance signal acting as a constant background. One of the challenges of the PR technique is to eliminate the PL contribution to the signal. Shen and Dutta ${ }^{29}$ proposed a sweeping photoreflectance (SPR) technique to minimize the effect of the PL during the measurements. Using their set-up as a model, the PR experimental set-up realized in our lab is presented in Figure 3.6. Light from a tungsten lamp is sent to a monochromator having a focal length of $1 / 4 \mathrm{~m}$ that will output a certain wavelength controlled via the PC interface. The output light from the monochromator will be focused on the sample with a lens forming a narrow spot $(0.5 \mathrm{~mm}$ width by $6 \mathrm{~mm}$ height), where the modulated laser light is also focused. The laser light is incident at a different angle to prevent its later collection in the detector used to monitor the reflected light. As the source for the coherent light, we used a semiconductor laser diode (Philips CQL806/30) combined with a constant power laser driver (Thorlabs LD1100) to supply the constant current. The detector placed at the reflection angle for the probe beam was protected with a bandpass filter to block the wavelength of scattered light corresponding to the laser wavelength. The detector is a silicon PIN photodiode detector that sends the signal to the lock-in amplifier for the $\mathrm{AC}$ signal detection and to a DC voltmeter via a V/A preamplifier (Ithaco 1641 preamplifier). The quantity of interest in the PR technique, $\Delta \mathrm{R} / \mathrm{R}$ is obtained through the normalization procedure realized by dividing $\mathrm{I}_{0} \Delta \mathrm{R}$ measured with the lock-in amplifier by $\mathrm{I}_{0} \mathrm{R}$ measured with the DC voltmeter, both signals being recorded and stored at the same time with the corresponding monochromator wavelength using the LabView data acquisition system.

In the sweeping technique, the mechanical chopper from the classical setup (Fig. 3.5) is replaced by an acousto-optic modulator/deflector which operates in the deflection mode (Fig. 3.6). The pump beam is swept on the sample with respect to the probe beam position. During half of its periodic oscillation, the pump beam spot will coincide with the probe beam spot to 


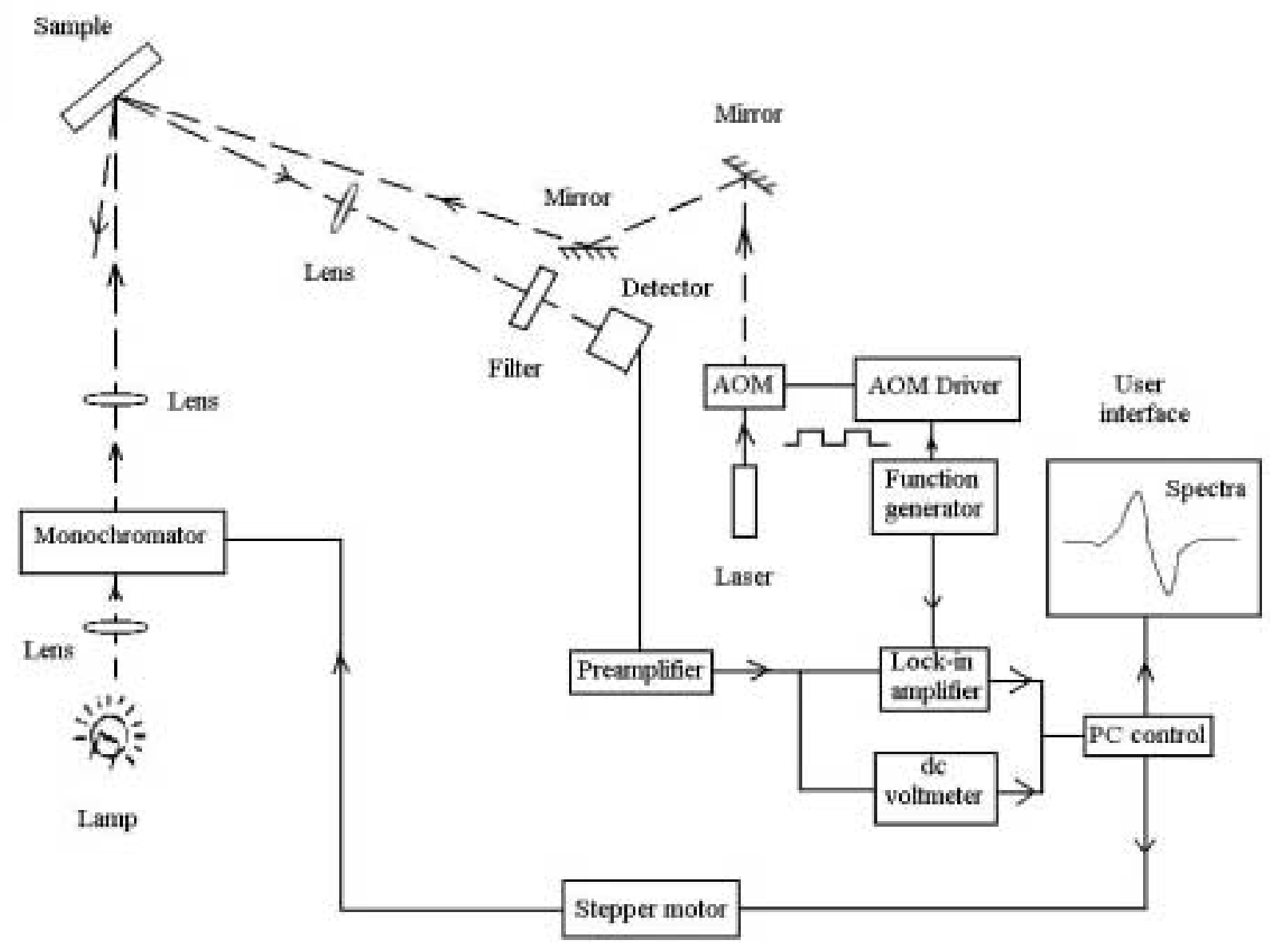

Figure 3.6 Schematic representation of the photoreflectance set-up. 
modulate the reflectance from the sample. The sweeping distance is kept smaller than the active area of the detector and the laser intensity is kept constant. If collected properly, the PL signal produced by the sweeping beam is seen by the detector as a DC luminescence $\mathrm{I}_{\mathrm{PL}}\left(\lambda_{\mathrm{PL}}\right)$. This way the detector will not see two-mixed AC signals but only the useful PR signal separated from the PL contribution. Moreover, the PL elimination was useful for the increase of the relative signalto-noise ratio. A few experiments were done with the acousto-optic modulator working in chopping mode to observe differences in PR signal amplitudes, but without observing any significant changes. In the chopping modulation case the PL signal was bigger as expected.

Each component of the set-up and its influence to the final PR signal was carefully checked and replaced if necessary. One of the components that influenced the noise in the detection scheme is the V/A preamplifier. Two types were investigated: an Oriel preamplifier (model 70710) and a Ithaco preamplifier (model 1641) with different amplification factors. At a certain wavelength the photodiode was kept in the dark chamber that was used for PC experiments without external illumination and the chopper supplied the AC reference signal. During a time period of $\sim 30 \mathrm{~min}$. the AC and DC signals detected by the lock-in and voltmeter were monitored to look at the mean value and standard deviation of the signals recorded. These signals represent the dark, or noise, AC and DC signal. The Ithaco preamplifier was preferred because of its higher capacity to detect AC signals for the same amplification scale while providing lower levels of noise due to the DC background signal.

To minimize the PL contribution to the lock-in signal while the PR contribution was increased, another set-up correction was performed by optimizing the system of lenses before the sample and after the reflection along the probe beam path. To collect the same solid angle of light at the detector as the one sent to the sample from the monochromator, the $\mathrm{L}_{1}$ and $\mathrm{L}_{2}$ corresponding focal numbers $\mathrm{n}_{1}=\mathrm{d}_{1} / \mathrm{f}_{1}$ and $\mathrm{n}_{2}=\mathrm{d}_{2} / \mathrm{f}_{2}$ need to be matched, where $\mathrm{d}_{1}$ and $\mathrm{d}_{2}$ are the corresponding lens diameters, and $f_{1}$ and $f_{2}$ are the corresponding lens focal lengths. This optimization was able to decrease the relative effect of the PL by almost an order of magnitude. At the same time an adequate diameter was chosen for the $\mathrm{L}_{2}$ lens to collect the sweeping PL generated by the laser in order to see it as a DC signal by keeping it in the active area of the detection photodiode. To check, the initial photodiode with a square active area of $16 \mathrm{~mm}^{2}$ was replaced with a photodiode having larger active area of $100 \mathrm{~mm}^{2}$ to see the influence on 
minimizing the PL signal. However, a considerable improvement has not been observed with enlarging the active area of the detector.

\subsection{Photoconductivity Set-up}

The potential of the spectral photoconductivity (SPC) characterization technique to study the photoconductor properties of semiconductors and to reveal imperfection states in the band gap $^{16}$ were the arguments to apply the SPC method in our lab. In the case of the GaN samples considered in our study, the PC signal is very small compared with the dark current. Detection of such weak photosignals needs a careful selection of several parameters. It is necessary to know the change in the resistance of the sample caused by incident radiation, the impedance of the measuring circuit, the response time, the capabilities and limitations of the photodetector, and sources of noise and their magnitudes. The combined knowledge of these factors helps in selecting the proper instruments. ${ }^{26}$

To resolve the weak photoconductivity signal in the case of epitaxial layers of GaN we have employed a modulation technique, using a mechanical chopper to modulate the incident light on the sample. The schematic of the SPC experiment is shown in Figure 3.7. The output signal is an AC signal mixed with the dark current. Even though very weak, the induced AC photosignal can be separated by a lock-in phase sensitive amplifier, having the same frequency as that of the chopper. The frequency of modulation of the light beam is determined in such a way as to match the response time of the photoconductor. In the case of GaN samples the response time was slow due to persistent photoconductivity, taking a long time to attain the steady state photocurrent. This is why the frequency for the type of samples we studied was chosen to be as low as possible, about 6-7 Hz. This lower limit was imposed by frequency nonhomogenity of the chopper that is seen by the amplifier at lower frequencies. The use of an appropriate frequency to maximize the signal needs to be done while considering at the same time the possibility of high $1 / \mathrm{f}$ noise. Four contacts were made at the corners of rectangular samples and the contacts were connected in pairs in such a way that the incident monochromatic light was focused between the two pairs. We have used soldered indium to make ohmic contacts on the n-type $\mathrm{GaN}$. Other p-type $\mathrm{GaN}$ samples had Ni/Au contacts deposited on them that 


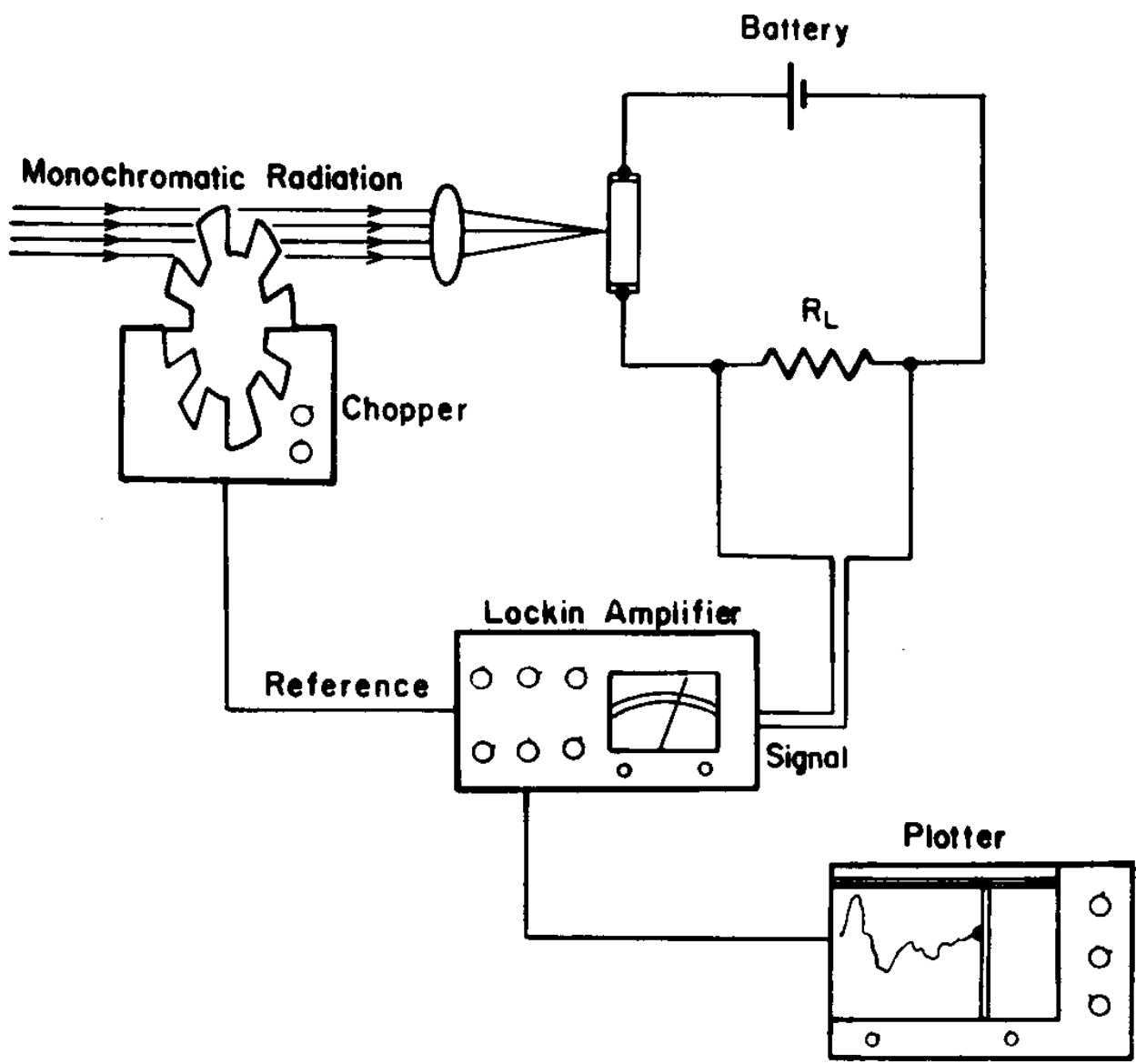

Figure 3.7 Phase-sensitive system for photoconductivity measurement. 
provided to be ohmic for this type of material. A load resistor was placed in series with the photoconductor being studied. The voltage was collected either from the sample or from the load resistor. As the voltage source we have used either a battery or a constant current source. Both cases provide the same relation of proportionality between the light generated photoconductivity and the voltages measured across the photoconductor as stated below.

In the case of the circuit with the battery providing a constant voltage, the photovoltage across the sample can be determined by evaluating the difference between the voltages with and without radiation: ${ }^{26}$

$$
V_{d}=\frac{R_{L} \mathrm{~V}}{\mathrm{R}_{\mathrm{L}}+R_{d}}
$$

When the sample is illuminated the voltage is:

$$
V_{I l l}=\frac{R_{L} \mathrm{~V}}{\mathrm{R}_{\mathrm{L}}+R_{I l l}}
$$

where $R_{d}$ is the dark resistance of the sample, $R_{I I l}$ is the illuminated sample resistance and $V$ is the applied voltage. The signal response is given by:

$$
\Delta V=V_{I l l}-V_{d}=R_{L} V\left[\frac{\Delta R}{\left(R_{L}+R_{I l l}\right)\left(R_{d}+R_{L}\right)}\right]
$$

where $\Delta \mathrm{R}=\mathrm{R}_{\mathrm{d}}-\mathrm{R}_{\mathrm{IIl}}$.

In the case when a constant current source is used to power the circuit, the photovoltage across the sample can be evaluated using a similar procedure to the one mentioned above.

The dark voltage is:

$$
V_{d}=\frac{R_{d} \mathrm{~V}}{\mathrm{R}_{\mathrm{L}}+R_{d}}
$$

and the voltage when the sample is illuminated is:

$$
V_{I l l}=\frac{R_{I l l} \mathrm{~V}}{\mathrm{R}_{\mathrm{L}}+R_{I l l}}
$$

Then the difference in conductance caused by the radiation is given by:

$$
\Delta G=\frac{1}{R_{I l l}}-\frac{1}{R_{d}}
$$

that will transform Equation 3.3 into: 


$$
\frac{\Delta V}{V}=\frac{R_{L} R_{d} R_{I l l} \Delta G}{\left(R_{L}+R_{I l l}\right)\left(R_{L}+R_{d}\right)}
$$

For the case when the change in the dark resistance is small $\left(\mathrm{R}_{\mathrm{d}} \approx \mathrm{R}_{\mathrm{III}}\right)$ as our samples case, the Equation 3.7 becomes:

$$
\frac{\Delta V}{V}=\frac{R_{L} R_{d}^{2} \Delta G}{\left(R_{L}+R_{d}\right)^{2}}
$$

which is the same as the Equation 2.10 from Reference 26, calculated for a constant voltage source. Equation 3.8, which assumes that the sample is illuminated uniformly, states the proportionality between the change in conductivity and the ratio between the measured DC and $\mathrm{AC}$ voltages. This equation will be applicable in case of either a constant voltage source or a constant current source.

The value of the load resistance $\mathrm{R}_{\mathrm{L}}$ that is controlled by the user can be optimized in order to obtain the maximum photoresponse. This will be obtained when the relation $\mathrm{d}(\Delta \mathrm{V}) / \mathrm{dR}_{\mathrm{L}}$ $=0$ is satisfied. The value for the photoresponse maximization will be $R_{L}=R_{d}$. Thus we used load resistors matched to our sample resistance.

Thus Equation 3.8 permits us to determine the spectral photoresponse experimentally under the assumption that the intensity of light on the sample remains constant over the wavelength range of the measurement, which is not a true statement for the tungsten lamp we have used. A lamp power calibration was done to eliminate the lamp power contribution to the photoresponse signal. For this purpose we replaced the sample circuit with a calibrated power meter borrowed from Prof. N.C. Giles' laboratory to measure the spectral power distribution of the entire system including the lamp, monochromator and optics.

Another particular problem with the SPC set-up was that the diffraction grating of the monochromator generates second order diffraction beams that are the doubled initial wavelength. This means that the first order diffraction on the monochromator grating of the long wavelengths are mixed together with the second order diffraction of the short wavelengths. To eliminate this problem we have used a set of bandpass filters, one cutting any wavelength below $300 \mathrm{~nm}$ to study the band gap response of the GaN samples that we measured, while for studying the states in the band gap, the second filter was necessary to cut all wavelengths below $570 \mathrm{~nm}$ that includes the second order diffraction of the short wavelengths. The two filters were considered, including their wavelength association, when doing the power calibration. As we will see in the 
next chapter, the power calibration influences the final SPC data and calibration curves from two different power meters were considered in the present study. 


\section{RESULTS}

\subsection{Concentration determination of small amounts of $\mathrm{Zn}$ in $\mathrm{Cd}_{1-\mathrm{x}} \mathrm{Zn}_{\mathrm{x}} \mathrm{Te}$ bulk semiconductors using photoreflectance spectroscopy}

As outlined in Section 2.1, PR spectroscopy has been demonstrated to be very effective for accurate determination of characteristic energy levels in compound semiconductors. The band gap energy in ternary or quaternary semiconductor compounds is dependent on the relative concentration of the constituents. It was proposed ${ }^{30}$ that the room temperature band gap energy of $\mathrm{Cd}_{1-\mathrm{x}} \mathrm{Zn}_{\mathrm{x}} \mathrm{Te}$ compounds can be estimated using PR with an accuracy of $0.4 \mathrm{meV}$ for small concentrations of $\mathrm{Zn}(\mathrm{x}<0.2)$, leading to a precision in composition determination of about 0.001 by way of band gap energy determination. Based on these data and on the research motivation presented in Section 1.3, the experiments presented here are focused on obtaining a high accuracy in $\mathrm{Cd}_{1-\mathrm{x}} \mathrm{Zn}_{\mathrm{x}} \mathrm{Te}$ compound concentration determination based on band gap estimation.

The first study included a set of twelve bulk $\mathrm{Cd}_{1-\mathrm{x}} \mathrm{Zn}_{\mathrm{x}} \mathrm{Te}$ samples with $0 \leq \mathrm{x} \leq 0.1$, which was furnished by eV-Products, Inc. The samples were grown using the vertical Bridgeman method and are considered to be high quality monocrystalline materials. The samples were $16 \times$ $16 \times 1 \mathrm{~mm}^{3}$ and most of them are oriented (211) or (111). This particular set of samples was used to obtain an estimation of the experimental precision for concentration determination. They were then sent to ChemIcon, Inc. as standards necessary for the calibration of their imaging technique as part of a larger, collaborative program.

The modulation of the samples to reveal the PR effect was done using a Phillips laser diode with a wavelength of $675 \mathrm{~nm}$. The energy corresponding to the emission line was above the band gap of materials studied and the output laser power was adjustable up to $30 \mathrm{~mW}$. The modulation frequency was kept at $200 \mathrm{~Hz}$. On each sample, five different spots were used to map the composition variation, four at the corners and one in the center. The wavelength ranges for the scans corresponded to the region were the band gap transition or excitonic transition should take place.

Various experimental parameters were optimized as described in Section 3.2 to obtain the maximum PR signal unperturbed by other effects such as PL or Franz-Keldysh oscillations. ${ }^{24}$ To 
avoid such perturbations and to optimize the maximum PR signal, a study of the laser output power influence on the signal was done on several samples. Some representative PR spectra for various laser powers are illustrated in Figure 4.1. It can be seen that with increasing the laser power the PL background also increases, a phenomenon that is not wanted. At the same time, the PR amplitude of the signal increases up to a laser power of $14.25 \mathrm{~mW}$, and then decreases for higher values. For the highest values of the laser power a feature is observed to the left of the main PR transition, corresponding to smaller energies, that can be attributed to either defects or impurities present in the material starting to contribute to the spectrum. The optimum power for which PL is sufficiently small and no "extra" feature is present to the left while the PR signal is maximized was chosen to be $9.5 \mathrm{~mW}$. This power was used for obtaining the PR spectrum of the entire set of eV-Products samples.

Values of $E_{g}$ as a function of concentration have to be extracted from a theoretical fit. The third derivative modulation theory presented in Section 2.1 is adequate for the experiments considered here, and the equation used to fit the measured spectra was deduced based on Equation 2.13:

$$
\Delta R / R=C \times\left[\left(E-E_{g}\right)^{2}+\Gamma^{2}\right]^{-m / 2} \times \cos (\phi-m \psi)
$$

where:

$$
\psi=\cos ^{-1}\left(\frac{E-E_{g}}{\sqrt{\left(E-E_{g}\right)^{2}+\Gamma^{2}}}\right)
$$

and the other parameters were explained in Section 2.1. The m value for the band gap fit is 2.5, while for an excitonic fit $\mathrm{m}=2$, as previously outlined. The experimental data were fit to Equation 4.1 using a non-linear least-squares fitting routine based on a modified MarquardtLevenberg algorithm. These fits were performed using SigmaPlot (Jandel Scientific). All experiments were done at room temperature and an excitonic contribution to the PR signal was expected. $^{15,30}$

An equation based on a prior study linking alloy composition with band gap ${ }^{30}$ was used to calculate the compound concentration from the band gap value (in $\mathrm{eV}$ ):

$$
\mathrm{E}_{\mathrm{g}}\left(\mathrm{Cd}_{1-\mathrm{x}} \mathrm{Zn}_{\mathrm{x}} \mathrm{Te}\right)=\mathrm{E}_{\mathrm{g}}(\mathrm{CdTe})+0.631 \mathrm{x}+0.128 \mathrm{x}^{2}
$$




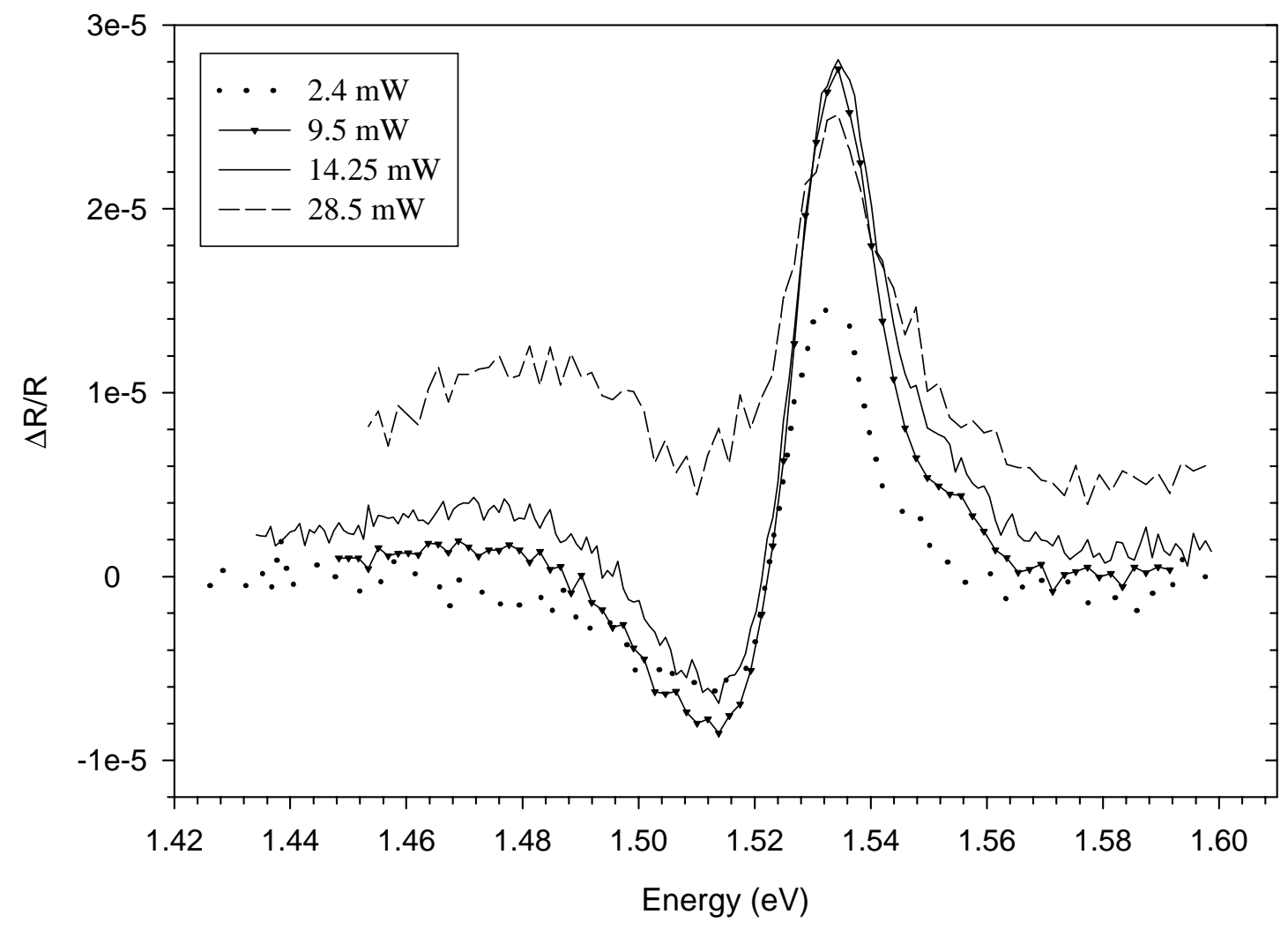

Figure 4.1 Output laser power influence on the photoreflectance signal for a $\mathrm{Cd}_{0.954} \mathrm{Zn}_{0.046} \mathrm{Te}$ sample (8222-T3). 
The first term gives the base band gap for CdTe with the other terms giving the change in $\mathrm{E}_{\mathrm{g}}$ with composition. Tobin et al. ${ }^{30}$ have used an $\mathrm{E}_{\mathrm{g}}$ for CdTe of $1.5045 \mathrm{eV}$ based on an interpretation of the PR signal as originating from a band-to-band transition. This conflicts with analysis of this transition by $\mathrm{Yu}$ et al. ${ }^{31}$ and Sanchez-Almazan et al. ${ }^{15}$ For each of the two $\mathrm{x}=0$ samples, the spectra from all five spots on each sample were fitted taking into account both band gap and excitonic effects. The exciton binding energy was fixed at $10 \mathrm{meV}$, as reported in literature. ${ }^{31}$ The fit results can be found in Table 4.1 together with all other fit results for the eVProducts twelve-sample set. A typical spectra for the CdTe samples is plotted in Figure 4.2, including the fit that adds the two relative contributions of the transitions considered. The band gap of CdTe was calculated as the mean value of all the measurements of the two samples. The average band gap energy was found to be $1.5145 \mathrm{eV}$, in very good agreement with the value of $1.514 \mathrm{eV}$ that is reported earlier. ${ }^{31}$ The value obtained for our $\mathrm{x}=0$ samples was used as the endpoint for the CdTe band gap energy in Equation 4.3 to compute the $\mathrm{x}$-value, which resulted in the following equation for $\mathrm{x}$ :

$$
x=-2.4648+\sqrt{7.8125 E_{g}-5.7562}
$$

where $\mathrm{E}_{\mathrm{g}}$ of the $\mathrm{Cd}_{1-\mathrm{x}} \mathrm{Zn}_{\mathrm{x}} \mathrm{Te}$ compound is measured in $\mathrm{eV}$.

Analysis of the PR spectra of the $\mathrm{Cd}_{1-\mathrm{x}} \mathrm{Zn}_{\mathrm{x}} \mathrm{Te}$ samples with $0<\mathrm{x}<0.1$ revealed that a single excitonic line fits the experimental results well (Figure 4.3) for the laser power chosen. $\Gamma$ broadening values resulting from the fits are below $20 \mathrm{meV}$ which indicates high quality material, so it was considered that excitonic effects are the main contribution to the PR spectra as also suggested in other work. ${ }^{15}$ The higher quality of material is associated with the presence of excitons and ultimately with their domination of the PR spectra. Band gap energies obtained from the PR fit results can be found in Table 4.1. The exciton binding energy of $10 \mathrm{meV}$ for $\mathrm{CdTe}$ was added to the excitonic transition energies to compute $\mathrm{E}_{\mathrm{g}}$ and determine the $\mathrm{Zn}$ concentration using Equation 4.4. The maps of the concentration variation for samples with $\mathrm{x}$ smaller than 0.1 are presented in Figure 4.4. Mean values for each of the sample are also indicated. The relative order of the mean values for composition are in good agreement with transmittance experiments on the same set of samples that was done in Professor L. Halliburton's laboratory. The transmittance spectra can be found in Figure 4.5, where it can be observed that the order in composition of samples deduced from PR corresponds to the same order in wavelengths for absorption revealed by transmittance experiments. 


\begin{tabular}{|c|c|c|c|c|c|c|c|}
\hline No. & File & Spot Position & $\begin{array}{c}\text { Modulation } \\
\text { method }\end{array}$ & $E_{g}(e V)$ & $\begin{array}{c}\Gamma_{1} \\
(\mathrm{meV})\end{array}$ & $\begin{array}{c}\Gamma_{2} \\
(\mathrm{meV})\end{array}$ & $\begin{array}{c}\text { Zn conc., } \\
x(\%)\end{array}$ \\
\hline 9061-10a & 110299a & Center & Sweep & 1.514 & 12 & 13.4 & 0 \\
\hline 9061-10a & $110299 b$ & Center & Chopped & 1.514 & 13.1 & 14.1 & 0 \\
\hline 9061-10a & $110299 \mathrm{c}$ & Center & Sweep & 1.514 & 15 & 14.9 & 0 \\
\hline $9061-10 a$ & 110299d & Upper, Right & Chopped & 1.5128 & 11.2 & 14.8 & 0 \\
\hline 9061-10a & $110299 \mathrm{e}$ & Upper, Right & Sweep & 1.5142 & 11.1 & 15.3 & 0 \\
\hline $9061-10 a$ & $110299 \mathrm{f}$ & Upper, Right & Sweep & 1.5156 & 12.9 & 16.2 & 0 \\
\hline $9061-10 a$ & $110299 \mathrm{~g}$ & Upper, Left & Sweep & 1.5149 & 10.3 & 14 & 0 \\
\hline 9061-10a & 110399a & Lower, Left & Sweep & 1.514 & 12.1 & 14 & 0 \\
\hline $9061-10 a$ & $110399 b$ & Lower, Right & Sweep & 1.514 & 12.1 & 14.5 & 0 \\
\hline 9061-10a & $110399 \mathrm{c}$ & Lower, Right & Chopped & 1.514 & 14.4 & 14.8 & 0 \\
\hline $9061-10 a$ & 110399d & Lower, Right & Chopped & 1.514 & 12.7 & 14.1 & 0 \\
\hline 9061-10a & 110999a & Center & Sweep & 1.514 & 12.4 & 14.2 & 0 \\
\hline $9061-10 a$ & $110999 b$ & Center & Sweep & 1.514 & 12.8 & 14.6 & 0 \\
\hline 9061-10a & 111099a & Upper, Right & Sweep & 1.514 & 12.4 & 14.2 & 0 \\
\hline 9061-10b & 111199a & Center & Sweep & 1.5148 & 15 & 17.3 & 0 \\
\hline $9061-10 \mathrm{~b}$ & 111299a & Upper, Right & Sweep & 1.5154 & 15.8 & 17.8 & 0 \\
\hline $9061-10 b$ & $111299 b$ & Upper, Left & Sweep & 1.5155 & 15.2 & 17.3 & 0 \\
\hline $9061-10 b$ & 111399a & Lower, Left & Sweep & 1.5156 & 14.4 & 17.2 & 0 \\
\hline $9061-10 b$ & $111399 b$ & Center & Sweep & 1.5156 & 14.8 & 17.3 & 0 \\
\hline $9061-10 b$ & $111599 \mathrm{c}$ & Lower, Right & Chopped & 1.5133 & 12.5 & 15.1 & 0 \\
\hline $9061-10 b$ & 111599d & Lower, Right & Sweep & 1.514 & 15.1 & 16.4 & 0 \\
\hline 9871-T3-2a & 112499a & Lower, Left & Sweep & 1.5449 & - & 17.6 & 4.8 \\
\hline 9871-T3-2a & $112499 b$ & Lower, Right & Sweep & 1.5471 & - & 16.1 & 5.1 \\
\hline 9871-T3-2a & $112499 \mathrm{c}$ & Center & Sweep & 1.5469 & - & 19.5 & 5.1 \\
\hline 9871-T3-2a & 112499d & Center & Sweep & 1.5463 & - & 18.8 & 5.0 \\
\hline 9871-T3-2a & $112599 a$ & Center & Chopped & 1.547 & - & 19 & 5.1 \\
\hline 9871-T3-2a & $112599 \mathrm{~b}$ & Upper, Right & Sweep & 1.5466 & - & 19.7 & 5.0 \\
\hline 9871-T3-2a & 112699a & Center & Sweep & 1.5467 & - & 19.4 & 5.1 \\
\hline
\end{tabular}




\begin{tabular}{|c|c|c|c|c|c|c|c|}
\hline No. & File & Spot Position & $\begin{array}{c}\text { Modulation } \\
\text { method }\end{array}$ & $E_{g}(\mathrm{eV})$ & $\begin{array}{c}\Gamma_{1} \\
(\mathrm{meV})\end{array}$ & $\begin{array}{c}\Gamma_{2} \\
(\mathrm{meV})\end{array}$ & $\begin{array}{c}\text { Zn conc., } \\
x(\%)\end{array}$ \\
\hline 10118-3a & 112799a & Center & Sweep & 1.5316 & - & 14.5 & 2.7 \\
\hline $10118-3 a$ & $117799 b$ & Upper, Right & Sweep & 1.5312 & - & 15 & 2.6 \\
\hline $10118-3 a$ & $112799 \mathrm{c}$ & Upper, Left & Sweep & 1.5313 & - & 14.6 & 2.7 \\
\hline 10118-3a & $112899 a$ & Lower, Left & Sweep & 1.5317 & - & 14.6 & 2.7 \\
\hline 10118-3a & $112899 b$ & Lower, Right & Sweep & 1.5316 & - & 14.7 & 2.7 \\
\hline 9759-19a & $112999 a$ & Center & Sweep & 1.5254 & - & 14.4 & 1.7 \\
\hline 9759-19a & $112999 b$ & Upper, right & Sweep & 1.5254 & - & 15.3 & 1.7 \\
\hline $9759-19 a$ & $112999 \mathrm{c}$ & Upper, Left & Sweep & 1.5252 & - & 14.4 & 1.7 \\
\hline $9759-19 a$ & 120799a & Lower, Left & Sweep & 1.5252 & - & 14.8 & 1.7 \\
\hline $9759-19 a$ & $120799 b$ & Lower, Right & Sweep & 1.5248 & - & 14.4 & 1.6 \\
\hline $9759-19 a$ & 120899a & Center & Sweep & 1.5252 & - & 14.5 & 1.7 \\
\hline 10118-4b & $120299 a$ & Center & Sweep & 1.5344 & - & 10.3 & 3.1 \\
\hline $10118-4 b$ & 121099a & Upper, Right & Sweep & 1.5356 & - & 11.4 & 3.3 \\
\hline $10118-4 b$ & $121099 b$ & Upper, Left & Sweep & 1.5348 & - & 9.3 & 3.2 \\
\hline $10118-4 b$ & $121699 a$ & Lower, Left & Sweep & 1.5345 & - & 9.9 & 3.2 \\
\hline $10118-4 b$ & $121699 b$ & Lower, Right & Sweep & 1.5352 & - & 10.5 & 3.3 \\
\hline $10118-4 b$ & $121799 a$ & Center & Sweep & 1.535 & - & 10.3 & 3.2 \\
\hline 9478-c3-18c & 121999a & Center & Sweep & 1.5429 & - & 14.4 & 4.5 \\
\hline $9478-c 3-18 c$ & $121999 b$ & Upper, Left & Sweep & 1.5438 & - & 14.2 & 4.6 \\
\hline 9478-c3-18c & 122199a & Upper, Right & Sweep & 1.5441 & - & 15.4 & 4.7 \\
\hline $9478-c 3-18 c$ & $122199 b$ & Upper, Right & Sweep & 1.5451 & - & 15.2 & 4.8 \\
\hline 9478-c3-18c & 010200a & Lower, Right & Sweep & 1.5441 & - & 14.5 & 4.7 \\
\hline $9478-c 3-18 c$ & 010200b & Lower, Left & Sweep & 1.5433 & - & 14.4 & 4.5 \\
\hline \begin{tabular}{|c|}
$9038-H 3-7$ \\
\end{tabular} & 012300c & Upper, Right & Sweep & 1.5329 & - & 17.8 & 2.9 \\
\hline 9038-H3-8 & 012300d & Upper, Left & Sweep & 1.5302 & - & 13.3 & 2.5 \\
\hline 9038-H3-10 & 012300e & Lower, Left & Sweep & 1.5305 & - & 14.8 & 2.5 \\
\hline 9038-H3-11 & $012400 \mathrm{~b}$ & Lower, Right & Sweep & 1.5309 & - & 14.5 & 2.6 \\
\hline 9038-H3-11 & 012400c & Center & Sweep & 1.5302 & - & 14.8 & 2.5 \\
\hline
\end{tabular}




\begin{tabular}{|c|c|c|c|c|c|c|c|}
\hline No. & File & Spot Position & $\begin{array}{c}\text { Modulation } \\
\text { method }\end{array}$ & $E_{g}(\mathrm{eV})$ & $\begin{array}{c}\Gamma_{l} \\
(\mathrm{meV})\end{array}$ & $\begin{array}{c}\Gamma_{2} \\
(\mathrm{meV})\end{array}$ & $\begin{array}{c}\text { Zn conc., } \\
x(\%)\end{array}$ \\
\hline $\mathbf{1 0 1 1 8 - 3}$ & $012700 \mathrm{a}$ & Upper, Right & Sweep & 1.5326 & - & 14.8 & 2.9 \\
\hline $10118-3$ & $012800 \mathrm{a}$ & Upper, Left & Sweep & 1.5327 & - & 15.1 & 2.9 \\
\hline $10118-3$ & $012800 \mathrm{~b}$ & Lower, Left & Sweep & 1.5323 & - & 15.2 & 2.8 \\
\hline $10118-4$ & $012800 \mathrm{c}$ & Lower, Right & Sweep & 1.5322 & - & 15.2 & 2.8 \\
\hline $10118-3$ & $013100 \mathrm{a}$ & Center & Sweep & 1.533 & - & 14 & 2.9 \\
\hline $\mathbf{9 4 7 8 - C 3 - 1 8}$ & $020100 \mathrm{a}$ & Upper, Right & Sweep & 1.5443 & - & 14.6 & 4.7 \\
\hline $9478-\mathrm{C3}-18$ & $020200 \mathrm{a}$ & Center & Sweep & 1.544 & - & 14 & 4.6 \\
\hline $9478-\mathrm{C3}-18$ & $020300 \mathrm{a}$ & Upper, Left & Sweep & 1.5435 & - & 14.4 & 4.6 \\
\hline $9478-\mathrm{C3}-18$ & $020300 \mathrm{~b}$ & Lower, Left & Sweep & 1.5427 & - & 15.2 & 4.4 \\
\hline $9478-\mathrm{C} 3-18$ & $020400 \mathrm{a}$ & Lower, Right & Sweep & 1.5444 & - & 14.9 & 4.7 \\
\hline $9478-\mathrm{C3}-18$ & $020400 \mathrm{~b}$ & Center & Sweep & 1.5431 & - & 14.2 & 4.5 \\
\hline 8222-T3 & $102299 \mathrm{a}$ & Upper, Right & Sweep & 1.5445 & 23.1 & 14.1 & 4.7 \\
\hline $8222-\mathrm{T3}$ & $102299 \mathrm{c}$ & Upper, Left & Sweep & 1.5444 & 23.5 & 14.2 & 4.7 \\
\hline $8222-\mathrm{T3}$ & $102599 \mathrm{a}$ & Lower, Right & Sweep & 1.5452 & 22.7 & 14.5 & 4.8 \\
\hline $8222-\mathrm{T3}$ & $102799 \mathrm{a}$ & Center & Sweep & 1.5433 & 23 & 14.1 & 4.5 \\
\hline $8222-\mathrm{T3}$ & $102799 \mathrm{c}$ & Upper, Right & Sweep & 1.5434 & 22 & 13.8 & 4.5 \\
\hline $8222-\mathrm{T3}$ & $102799 \mathrm{c}$ & Upper, Right & Sweep & 1.5429 & - & 14.4 & 4.5 \\
\hline $8222-\mathrm{T3}$ & $102799 \mathrm{~d}$ & Upper, Right & Chopped & 1.5433 & 22.6 & 13.9 & 4.5 \\
\hline $8222-\mathrm{T3}$ & $102799 \mathrm{~d}$ & Upper, Right & Chopped & 1.5428 & - & 14.2 & 4.4 \\
\hline A57-18b & $050900 \mathrm{f}$ & Center & Sweep & 1.5790 & 8.2 & 11.2 & 10.0 \\
\hline A57-18c & $051000 \mathrm{~b}$ & Center & Sweep & 1.5785 & 8.1 & 11 & 10.0 \\
\hline
\end{tabular}

Table 4.1 Results of fitting the PR spectra for the eV-Products samples obtained using an output laser power of $9.5 \mathrm{~mW} . \Gamma_{1}$ and $\Gamma_{2}$ represent the broadening parameters for band gap transition and excitonic transition, respectively. 


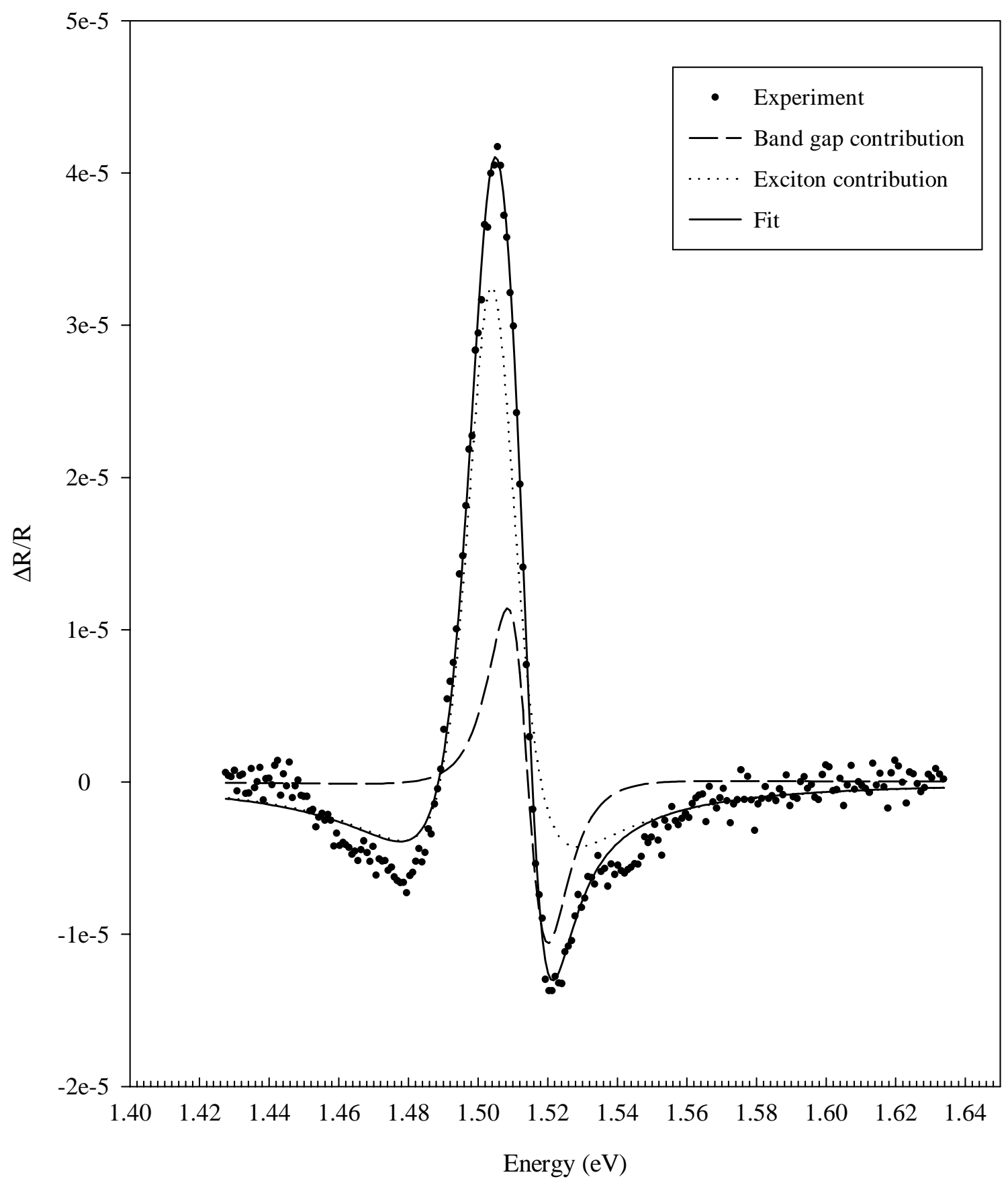

Figure 4.2 Double transition fit to the CdTe 9061-10a sample featuring the excitonic and band gap contributions to the PR spectrum. 


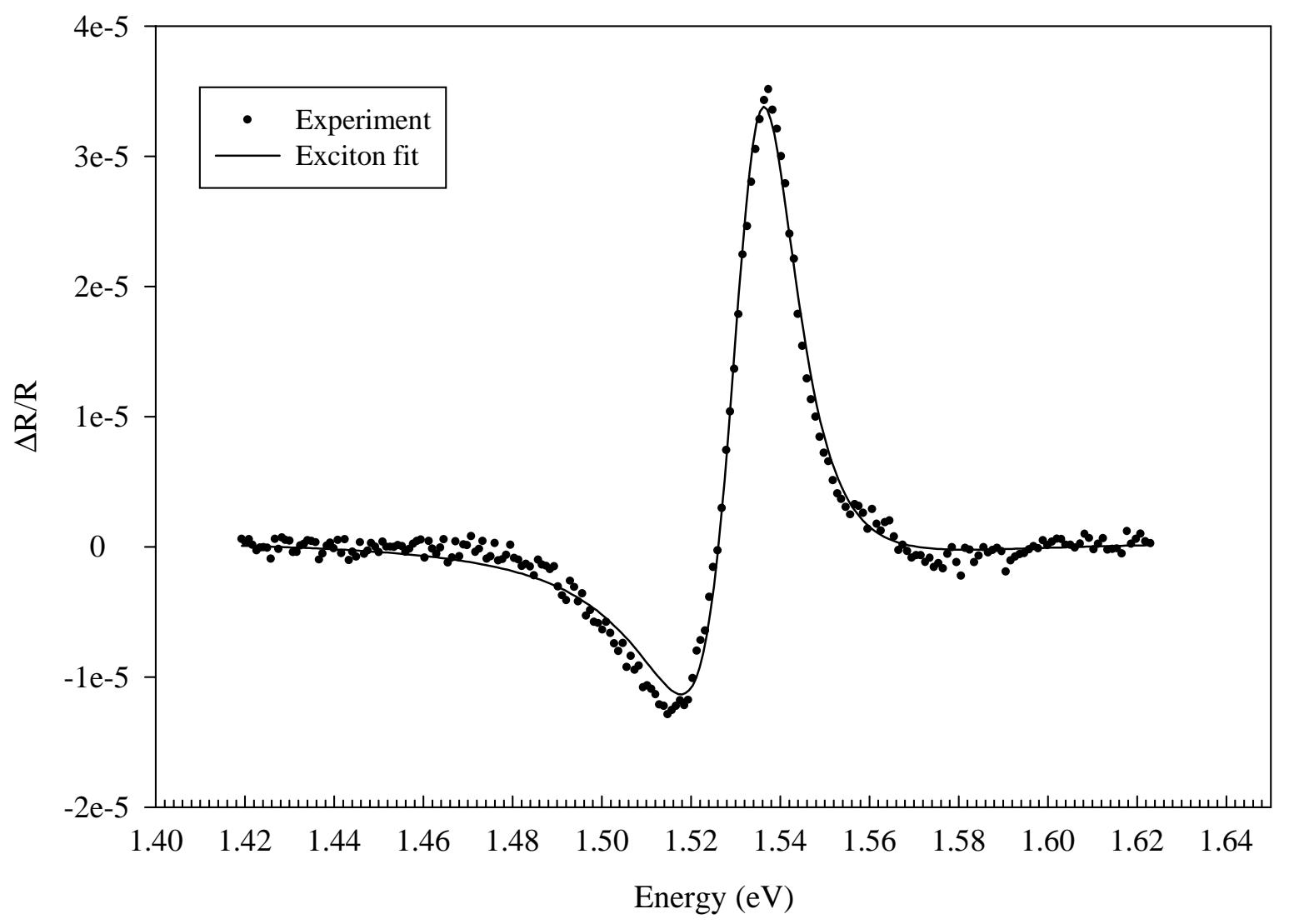

Figure 4.3 Representative PR spectra for all $\mathrm{Cd}_{1-\mathrm{x}} \mathrm{Zn}_{\mathrm{x}} \mathrm{Te}$ eV-Products samples with $\mathrm{x}<0.1$, including the single excitonic transition fit (this is sample 9478-C3-18c with $\mathrm{x}=0.046$ ). 

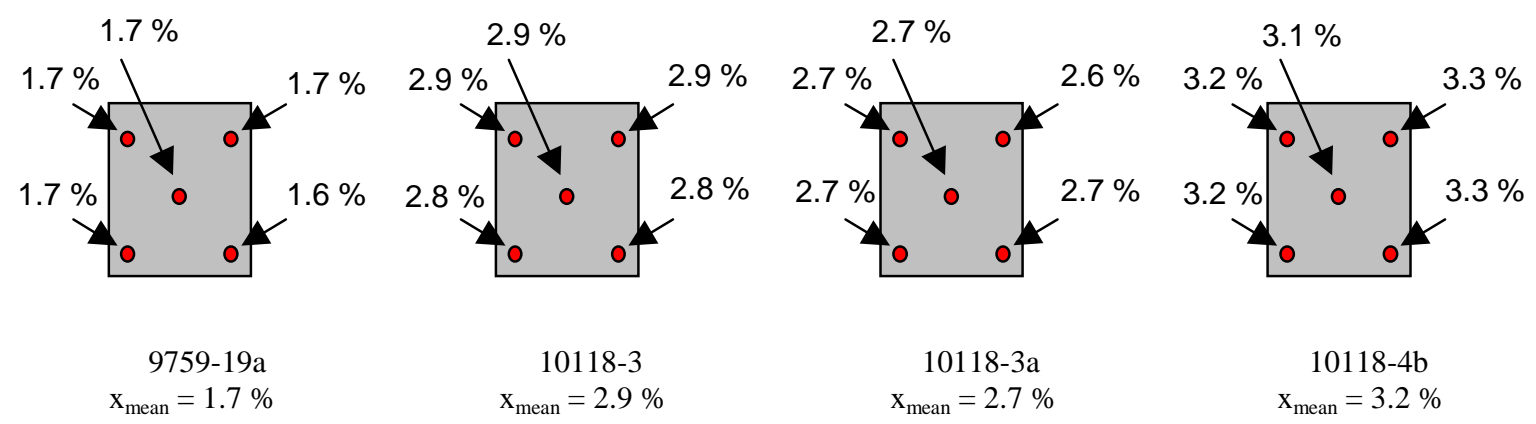

$$
\begin{gathered}
10118-3 \\
\mathrm{x}_{\text {mean }}=2.9 \%
\end{gathered}
$$

$$
\begin{gathered}
10118-3 \mathrm{a} \\
\mathrm{x}_{\text {mean }}=2.7 \%
\end{gathered}
$$

10118-4b

$$
\mathrm{x}_{\text {mean }}=3.2 \%
$$
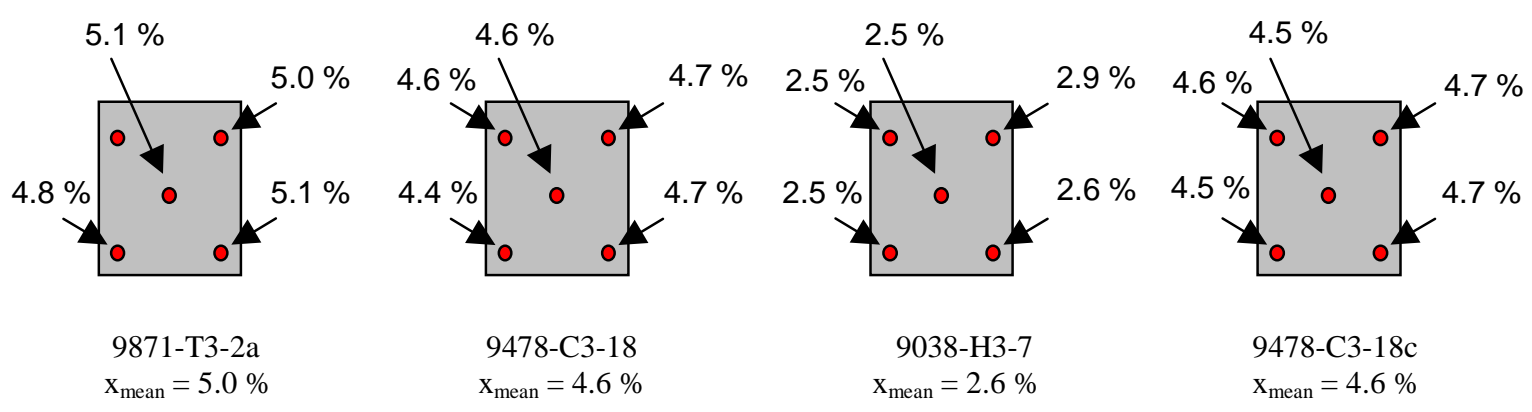

9478-C3-18c

$\mathrm{x}_{\text {mean }}=4.6 \%$

Figure 4.4 Compositional variation on the surface of the eV-Products $\mathrm{Cd}_{1-\mathrm{x}} \mathrm{Zn}_{\mathrm{x}} \mathrm{Te}$ samples with $0<\mathrm{x}<0.1$. 


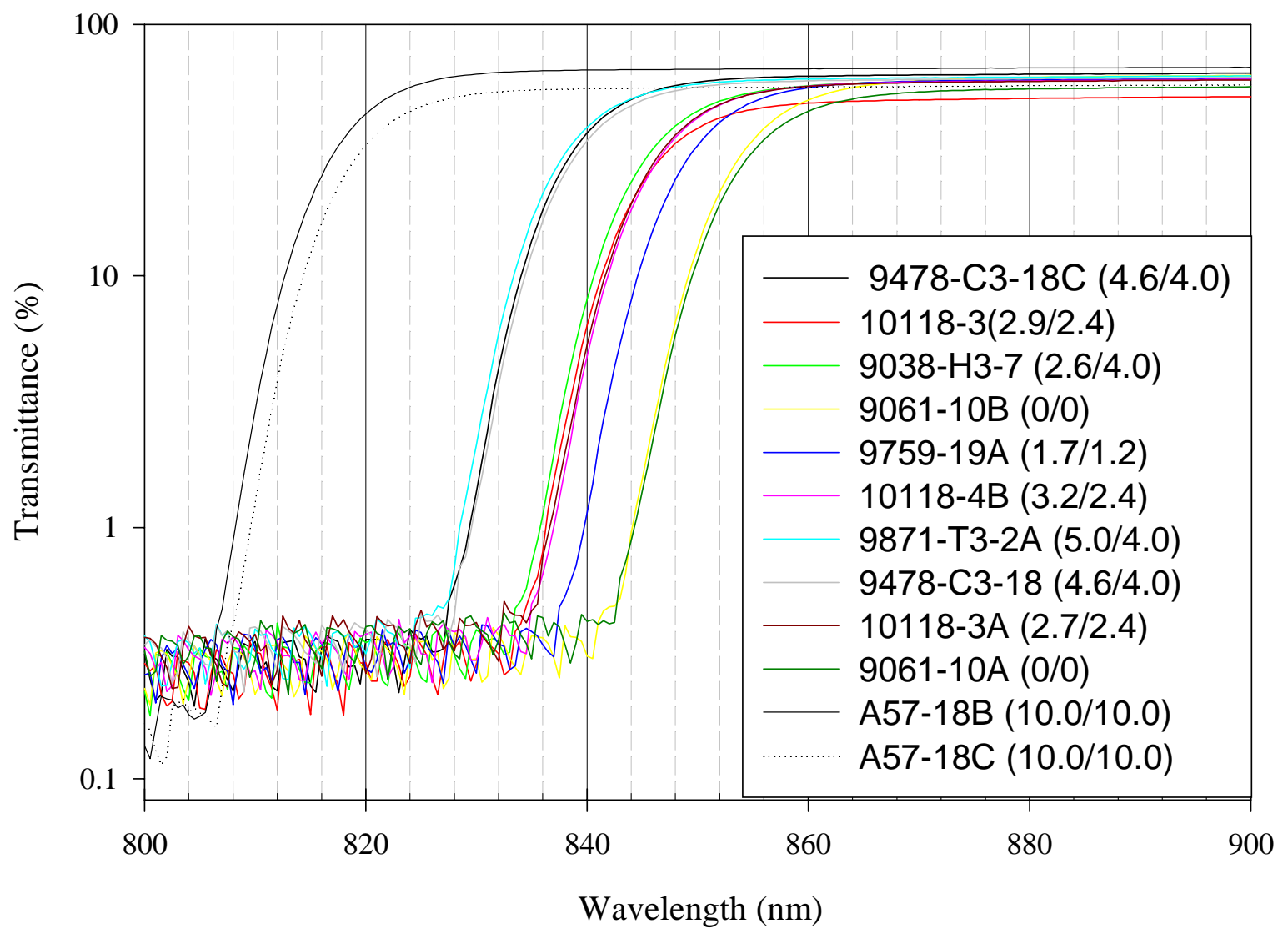

Figure 4.5 Experimental transmittance data for the eV-Products sample set. In parenthesis are the calculated $\mathrm{Zn}$ concentrations from PR fits and the values furnished by eV-Products, respectively. 
The eV-Products samples supposed to have $10 \%$ of $\mathrm{Zn}$ in composition cannot be fitted with excitonic line shape only and again the double contribution transition fit was used with the excitonic contribution being dominant. The calculated relative $\mathrm{Zn}$ concentrations from the band gap energy are $10.0 \%$, which is exactly the same as the ones provided by the crystal growers. A PR spectrum is represented in Figure 4.6. Both the two transitions fit and the excitonic only fit are plotted. The two-transition fit is better despite the other imperfections of the spectra that can be found at higher and lower energies than the transition. To the right of the PR transition the Franz-Keldysh oscillations can be observed which could not be reduced by lowering the laser power. This is considered to be an indication that this oscillation effect is not dependent on the intensity of the laser light but on the strength of the built-in surface electric field. This indicates that we are in the high field regime, which requires a more complicated analysis. The feature at lower energies that is not well fit is believed to correspond to the perturbation of the excitonic transition, and its contribution can be reduced by laser power adjustment.

The estimation of error in the concentration determination is a delicate matter and will depend more on the accuracy of the model used to fit the PR spectra than on experiment uncertainties and the reproducibility of the data. The experimental reproducibility was studied using both sweeping and chopping PR techniques and sample measurement repetition. The experimental measurement uncertainties are small when compared with the fit issues. It is supposed that the value for the band gap energy providing the composition will be determined more accurately if the spectrum exhibits only one transition. In this case better precision in the determination of the band gap energy can be obtained since the number of fitting parameters is smaller and their values can be more easily controlled by physics theory. Once the uncertainty of 
the band gap determination is known, the uncertainty in composition determination can be calculated using Equation 4.4.

Another poorer quality material was considered in the PR analyses of the $\mathrm{Cd}_{1-\mathrm{x}} \mathrm{Zn}_{\mathrm{x}} \mathrm{Te}$ composition determination. The area of the sample was $11 \times 9 \times 0.3 \mathrm{~cm}^{3}$ but the sample was broken into four smaller pieces. Figure 4.7 shows the sample, including the composition mapping done by using the PR method. Table 4.2 contains the PR parameters obtained from the fits. For most of the experimental spectra obtained on the surface of this sample the double transition is easily distinguished as illustrated in Figure 4.8. However, a particular region of the 


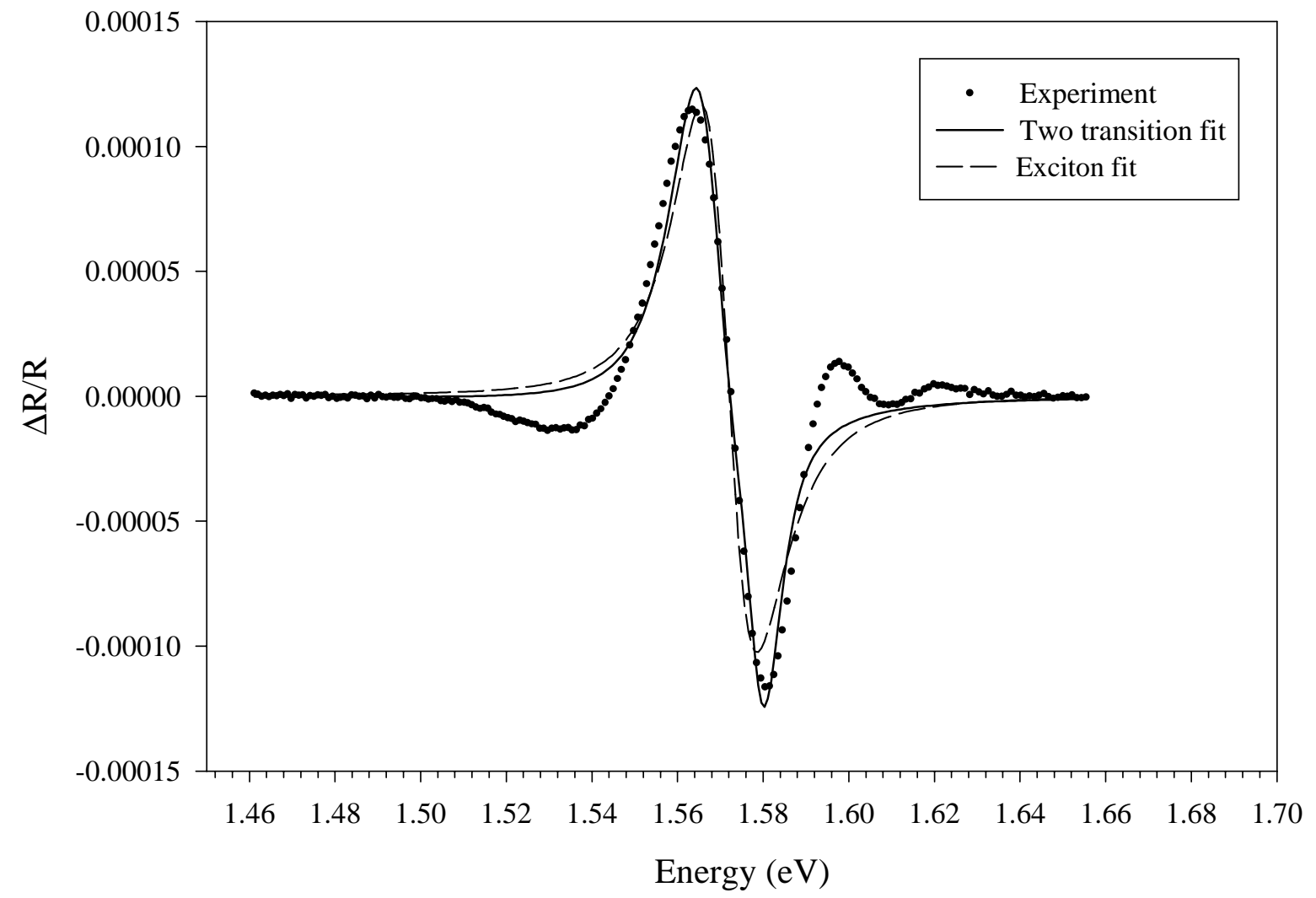

Figure 4.6 A typical PR spectrum and its fit attempts for the case of $10 \% \mathrm{Zn}$ eV-Products samples (sample A57-18c). 


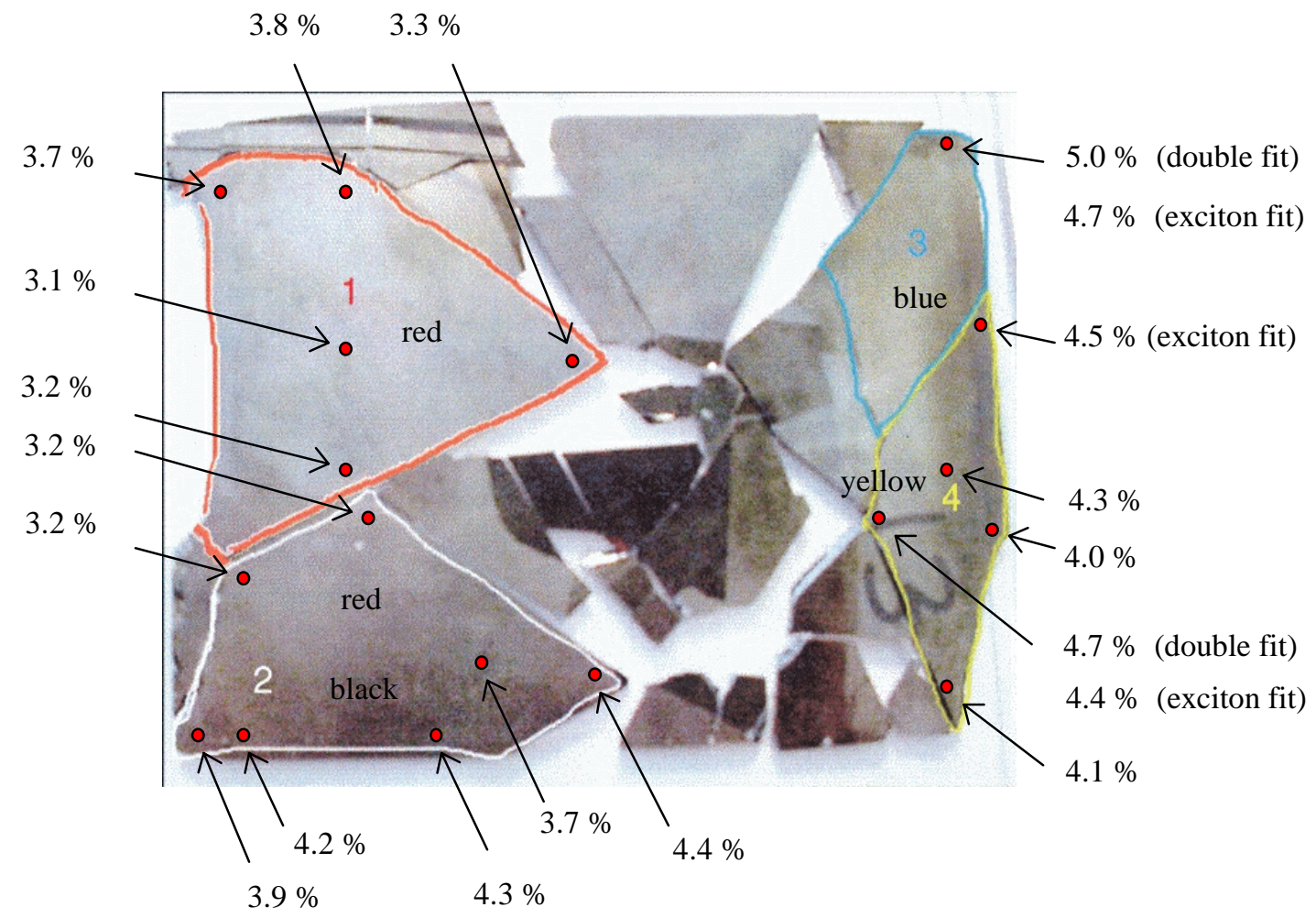

Figure 4.7 A large $\left(11 \times 9 \times 0.3 \mathrm{~cm}^{3}\right) \mathrm{Cd}_{1-\mathrm{x}} \mathrm{Zn}_{\mathrm{x}}$ Te sample, mapped in composition along its surface using the PR technique. Exciton only fits are indicated. 


\begin{tabular}{|c|c|c|c|c|c|c|c|}
\hline No. & Fragment & Spot Position & $\begin{array}{c}\text { Imaging } \\
\text { Colors }\end{array}$ & $E_{g}(\mathrm{eV})$ & $\begin{array}{c}\Gamma_{1} \\
(\mathrm{meV})\end{array}$ & $\begin{array}{c}\Gamma_{2} \\
(\mathrm{meV})\end{array}$ & $\begin{array}{c}\text { Zn conc., } \\
X(\%)\end{array}$ \\
\hline $010300 \mathrm{~d}$ & 4 & lower & yellow & 1.5403 & 12.2 & 15.2 & 4.1 \\
\hline $010400 \mathrm{a}$ & 4 & center - right & yellow & 1.5396 & 19.5 & 15.8 & 4.0 \\
\hline $010400 \mathrm{~b}$ & 4 & center - right & yellow & 1.5399 & 14.3 & 16.7 & 4.1 \\
\hline $010500 \mathrm{a}$ & 4 & center - right & yellow & 1.5388 & 13.7 & 14.6 & 3.9 \\
\hline $010600 \mathrm{~b}$ & 4 & center - left & yellow & 1.5440 & 20.5 & 18.8 & 4.7 \\
\hline $010600 \mathrm{~b}$ & 4 & center - left & yellow & 1.5420 & - & 18.6 & 4.4 \\
\hline $011200 \mathrm{a}$ & 4 & upper & yellow & 1.5430 & - & 23.0 & 4.5 \\
\hline $011100 \mathrm{a}$ & 4 & center & yellow & 1.5416 & 21.4 & 17.5 & 4.3 \\
\hline $011500 \mathrm{~b}$ & 3 & upper & blue & 1.5461 & 25.3 & 18.6 & 5.0 \\
\hline $011500 \mathrm{~b}$ & 3 & upper & blue & 1.5440 & - & 18.8 & 4.7 \\
\hline $011700 \mathrm{a}$ & 2 & right - edge & red, black & 1.5422 & 17.8 & 16.8 & 4.4 \\
\hline $011700 \mathrm{~b}$ & 2 & center & red & 1.5378 & 13.3 & 15.6 & 3.7 \\
\hline $011800 \mathrm{a}$ & 2 & left - edge & red & 1.5343 & 13.5 & 15.7 & 3.2 \\
\hline $011900 \mathrm{c}$ & 2 & lower & black & 1.5414 & 17.7 & 15.1 & 4.3 \\
\hline $012200 \mathrm{a}$ & 1 & upper - left & red, black & 1.5379 & 14.5 & 15.4 & 3.7 \\
\hline $012200 \mathrm{~b}$ & 1 & upper - right & red & 1.5385 & 15.9 & 15.3 & 3.8 \\
\hline $012300 \mathrm{a}$ & 1 & lower & red & 1.5346 & 13.2 & 16.0 & 3.2 \\
\hline $012300 \mathrm{~b}$ & 1 & center-right & red & 1.5352 & 12.0 & 16.2 & 3.3 \\
\hline
\end{tabular}

Table 4.2 PR fit results for the large $\left(11 \times 9 \times 0.3 \mathrm{~cm}^{3}\right) \mathrm{Cd}_{1-\mathrm{x}} \mathrm{Zn}_{\mathrm{x}} \mathrm{Te}$ sample corresponding to different spots on the sample. 


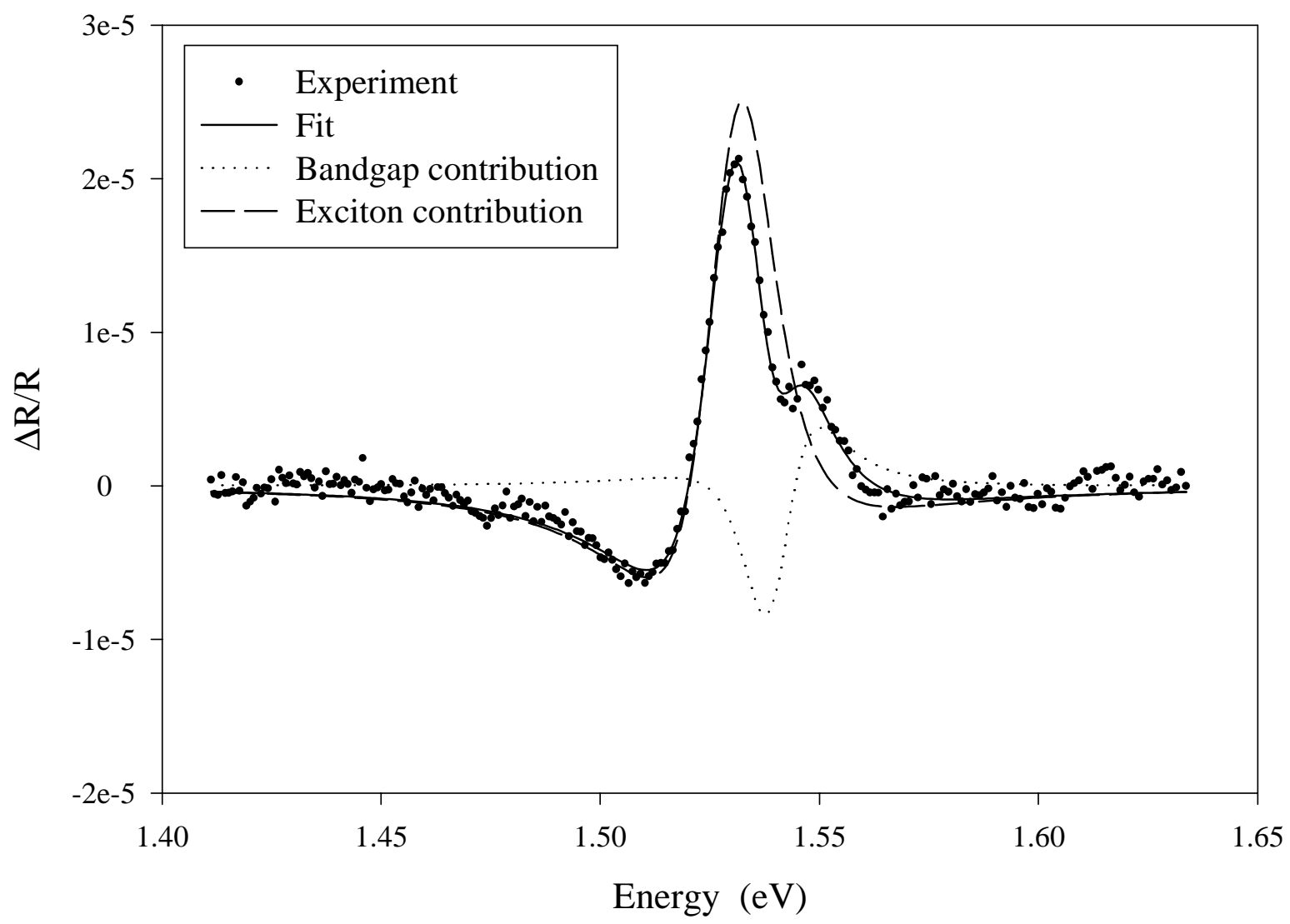

Figure 4.8 Typical double transition PR spectrum and fit for large $\left(11 \times 9 \times 0.3 \mathrm{~cm}^{3}\right) \mathrm{Cd}_{1-\mathrm{x}} \mathrm{Zn}_{\mathrm{x}} \mathrm{Te}$ sample, featuring the excitonic and band gap contributions to the fit. 
sample (the upper-right portion in Fig. 4.7) is observed to provide spectra that are believed to be only excitonic in nature. One of these spectra and its fit can be found in Figure 4.9. Taking into account the large area of the sample and supposing that inhomogeneities can easily occur, it is believed that this region contains higher quality material. The explanation for this effect can be attributed to a better local polishing resulting in a smoother surface or to sample growth issues such as an optimized growth for that particular region.

\subsection{Photoconductivity spectroscopy studies of epitaxially grown GaN using various growth methods}

The defect states in the forbidden gap of $\mathrm{GaN}$ are usually studied by luminescence measurements giving information about the defect state participating in the radiative recombination processes. Spectral photoconductivity (SPC) analysis can provide valuable information about the defect or impurity states and also the near band edge transitions participating in the absorption of light. Moreover, the SPC technique is capable of extracting useful information about the defect/impurity density of state distribution in the band gap. ${ }^{32}$

A set of GaN samples grown by rf-plasma MBE in our lab on sapphire substrates was selected for the SPC experiments. The samples were squares of $13 \times 13 \mathrm{~mm}^{2}$ and the epitaxiallygrown GaN layers were typically a few $\mu \mathrm{m}$ thick. Thickness values for each sample from transmittance experiments can be found in Table 4.3. These samples exhibit a wide range of optical and electrical properties as can be observed in the same table. All of the samples have ntype conductivity and are unintentionally doped. ${ }^{33}$ Several of the samples were of high electrical and structural quality, as indicated by their high mobility and low carrier concentration. Persistent photoconductivity (PPC) effects were observed in these samples and the analyses of the PPC data can be found in a prior publication. ${ }^{34}$ The reflectance and transmittance measurements were done in Prof. L.E. Halliburton lab and the photoluminescence experiments in Prof. N.C. Giles lab.

To obtain the material spectral photoresponse when using the experimental set-up described in Section 3.2, it is necessary to divide the ratio of the measured DC and AC voltages by the spectral power distribution of the light that is used to excite the sample. 


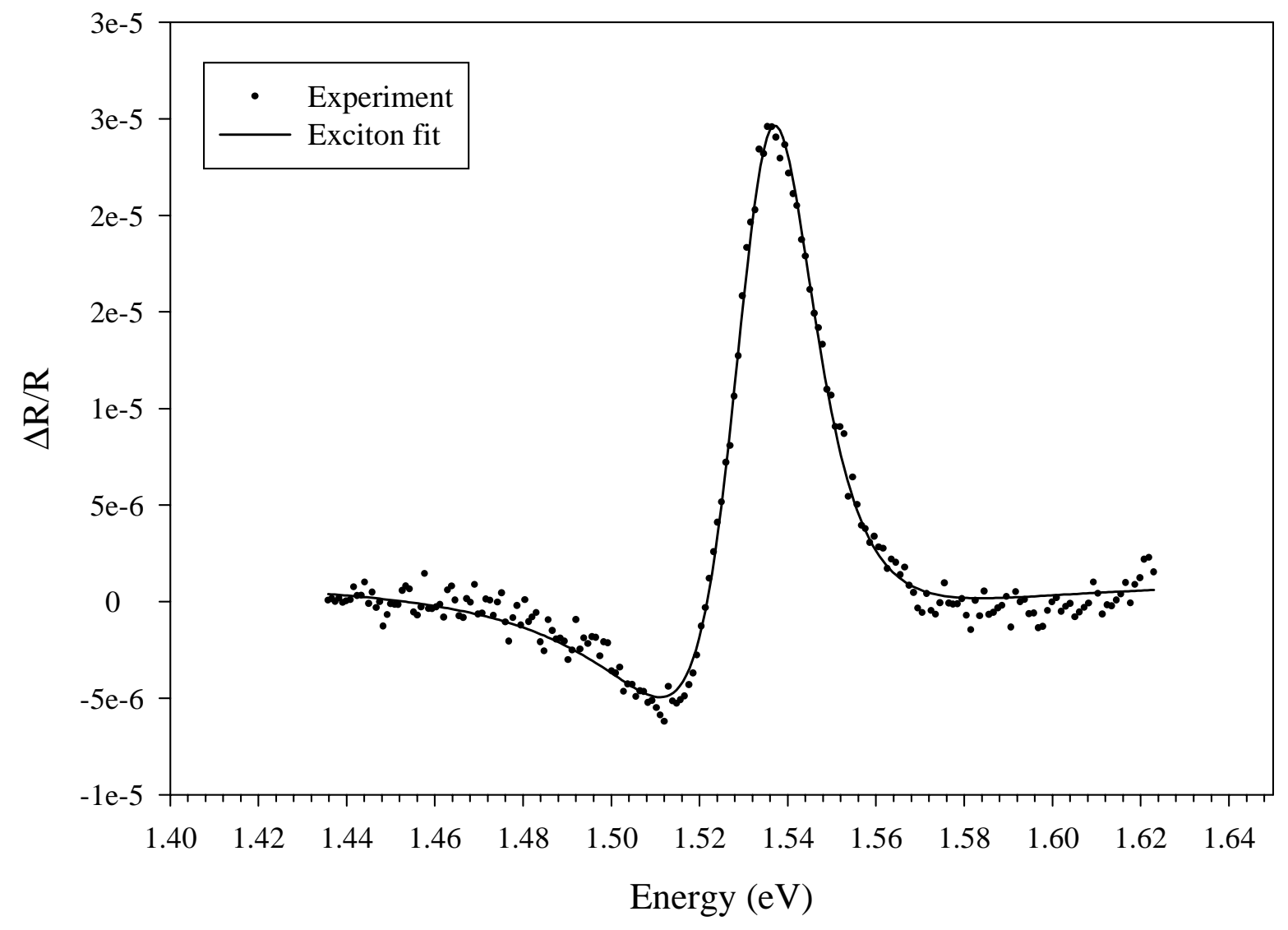

Figure 4.9 Single transition excitonic PR experiment and fit for the large $\left(11 \times 9 \times 0.3 \mathrm{~cm}^{3}\right)$ $\mathrm{Cd}_{1-\mathrm{x}} \mathrm{Zn}_{\mathrm{x}} \mathrm{Te}$ sample in the upper-right region of Fig. 4.7, where the material quality is considered to be higher. 


\begin{tabular}{|c|c|c|c|c|c|c|c|}
\hline $\begin{array}{c}\text { Sample } \\
\text { (Thickness) } \\
(\mu \mathrm{m})\end{array}$ & $\begin{array}{c}\tau_{50 \%} \\
\tau_{10 \%} \\
(\mathrm{~s})\end{array}$ & $\begin{array}{c}\tau_{\text {short }} \\
\left(10^{-3} \mathrm{~s}\right)\end{array}$ & $\begin{array}{c}\text { a-H } \\
\text { during } \\
\text { growth }\end{array}$ & $\begin{array}{c}\text { Yellow } \\
\text { PL }\end{array}$ & $\begin{array}{l}\text { Mobility } \\
\left(\mathrm{cm}^{2} / \mathrm{Vs}\right)\end{array}$ & $\begin{array}{c}\mathrm{N} \\
\text { Conc. } \\
\left(10^{16} \mathrm{~cm}^{-3}\right)\end{array}$ & $\begin{array}{c}\text { Exciton } \\
\text { in RT } \\
\text { Reflectance }\end{array}$ \\
\hline $\begin{array}{l}9824 \\
(9.2)\end{array}$ & $\begin{array}{l}4.0 \\
246\end{array}$ & 0.7 & & weak & 202 & 1.7 & $\begin{array}{c}\text { yes } \\
\text { strong }\end{array}$ \\
\hline $\begin{array}{l}9821 \\
(2.0) \\
\end{array}$ & $\begin{array}{l}4.8 \\
622 \\
\end{array}$ & 0.5 & & weak & 15 & 9.2 & no \\
\hline $\begin{array}{l}9826 \\
(7.5)\end{array}$ & $\begin{array}{c}5.1 \\
4,044\end{array}$ & 1.2 & & weak & 9.0 & 1.8 & yes \\
\hline $\begin{array}{l}9825 \\
(2.6)\end{array}$ & $\begin{array}{c}7.0 \\
7,171 \\
\end{array}$ & 1.4 & & N.D. & 12 & 1.7 & yes \\
\hline $\begin{array}{c}9910 \\
(1.72)\end{array}$ & $\begin{array}{c}13 \\
29,521\end{array}$ & 1.2 & & weak & 330 & 0.10 & yes \\
\hline $\begin{array}{l}9671 \\
(1.0)\end{array}$ & $\begin{array}{c}64 \\
6,704\end{array}$ & 2.3 & yes & moderate & 120 & 77 & no \\
\hline $\begin{array}{c}9730 \\
(0.98)\end{array}$ & $\begin{array}{c}214 \\
21,061 \\
\end{array}$ & 2.3 & yes & N.D. & 4 & 7.2 & no \\
\hline $\begin{array}{c}9710 \\
(0.85)\end{array}$ & $\begin{array}{c}293 \\
32,564\end{array}$ & 1.8 & yes & weak & 50 & 85 & no \\
\hline $\begin{array}{l}9823 \\
(2.1)\end{array}$ & $\begin{array}{c}308 \\
8,703\end{array}$ & 1.6 & yes & weak & 232 & 140 & yes \\
\hline $\begin{array}{l}9822 \\
(2.1)\end{array}$ & $\begin{array}{c}357 \\
52,922 \\
\end{array}$ & 1.0 & & moderate & 13 & 0.67 & (?) \\
\hline
\end{tabular}

Table 4.3 Various properties of some GaN samples grown by rf-plasma MBE included in this investigation. 
Therefore, the influence of the spectral power calibration on the final photoresponse curves was carefully considered. Figure 4.10 illustrates the power variation versus energy corresponding to the radiation used to photoexcite the samples. The two curves correspond to two different power meters and slightly different optical alignments used to perform the same experiment. In the lower energy range we can observe two main peaks, with the lower energy peak corresponding to the maximum intensity light emission from the lamp and the higher energy peak resulting from the blaze of the diffraction grating of the monochromator. It can be observed that the relative ratio of the two main peaks is changed for the two curves with the lower energy peak intensity larger in the case of the second power calibration curve. This phenomenon that can be explained by a combination of the replacement of the tungsten lamp bulb and the optimization process to maximize the lamp image on the monochromator diffraction grating. More importantly, the second power meter was a newer model (Coherent, Laser Mate- $\mathrm{Q}^{\mathrm{TM}}$ ) than the first (Coherent, model 212) with a vastly improved spectral sensitivity correction factor. Note that the kinks observed at energies $>3 \mathrm{eV}$ on the first power meter curve are eliminated by the better correction of the sensitivity factor in the case of second power meter. This precludes these artifacts from being interpreted as a feature in measured SPC spectrum.

First, the samples from Table 4.3 were measured in the SPC setup using the first power calibration curve and a $9 \mathrm{~V}$ battery as a constant voltage source to collect the photoexcited carriers. Some of the samples $(9730,9710,9823,9822)$ had a too small signal to noise ratio for performing the SPC measurement. Equation 2.13 corrected for the incident photon flux was used to calculate the photoconductance signal variation as a function of energy of the incident light:

$$
\Delta \mathrm{G}(\mathrm{h} v) \sim \Delta \mathrm{V} / \mathrm{V} / \mathrm{P}
$$

where $\mathrm{P}$ is the incident power, $\mathrm{h} v$ is the energy of the incident light, and the other parameters are explained in Section 3.2. The photoresponse curves are shown in Figure 4.11 and the spectra were normalized to the photoresponse above the band gap of $\mathrm{GaN}$, but they were not normalized for the sample thickness. Samples 9826 and 9824 were significantly thicker than the others (7.5 and $9.2 \mu \mathrm{m}$ vs 1 to $2 \mu \mathrm{m}$ ), which resulted in a much stronger below-gap signal.

The proposed SPC study tried to reveal defect or impurity-related states in the band gap participating in photoconductive processes, below the band edge transition. A discussion of the SPC data obtained on the samples from Table 4.3 can be found elsewhere. ${ }^{34}$ 


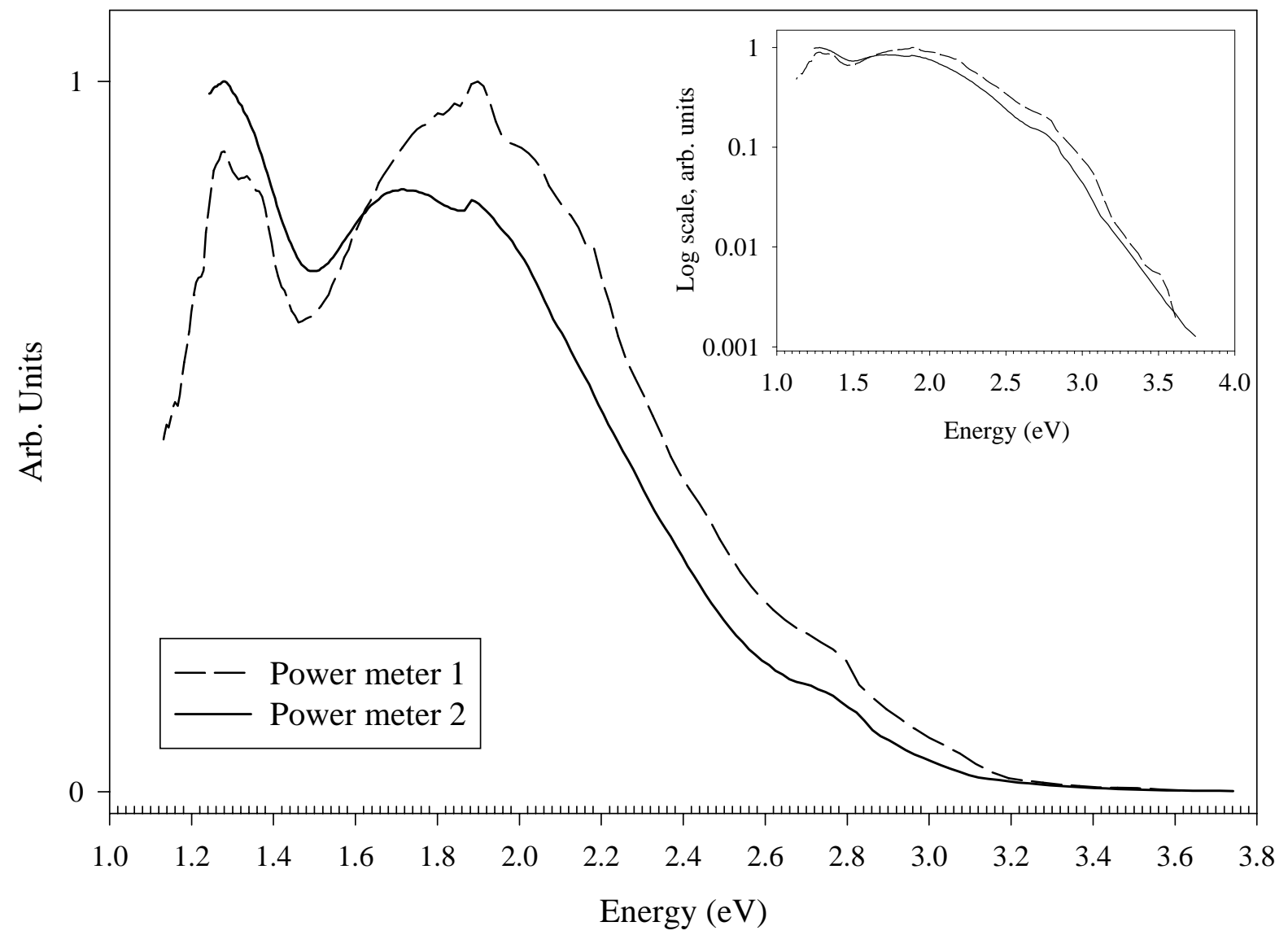

Figure 4.10 Power calibration curves for two and experimental set-ups. The inset represents the same result on a log scale. Power meter 1 corresponds to Coherent model 212 and power meter 2 to Coherent Laser Mate- $\mathrm{Q}^{\mathrm{TM}}$. 


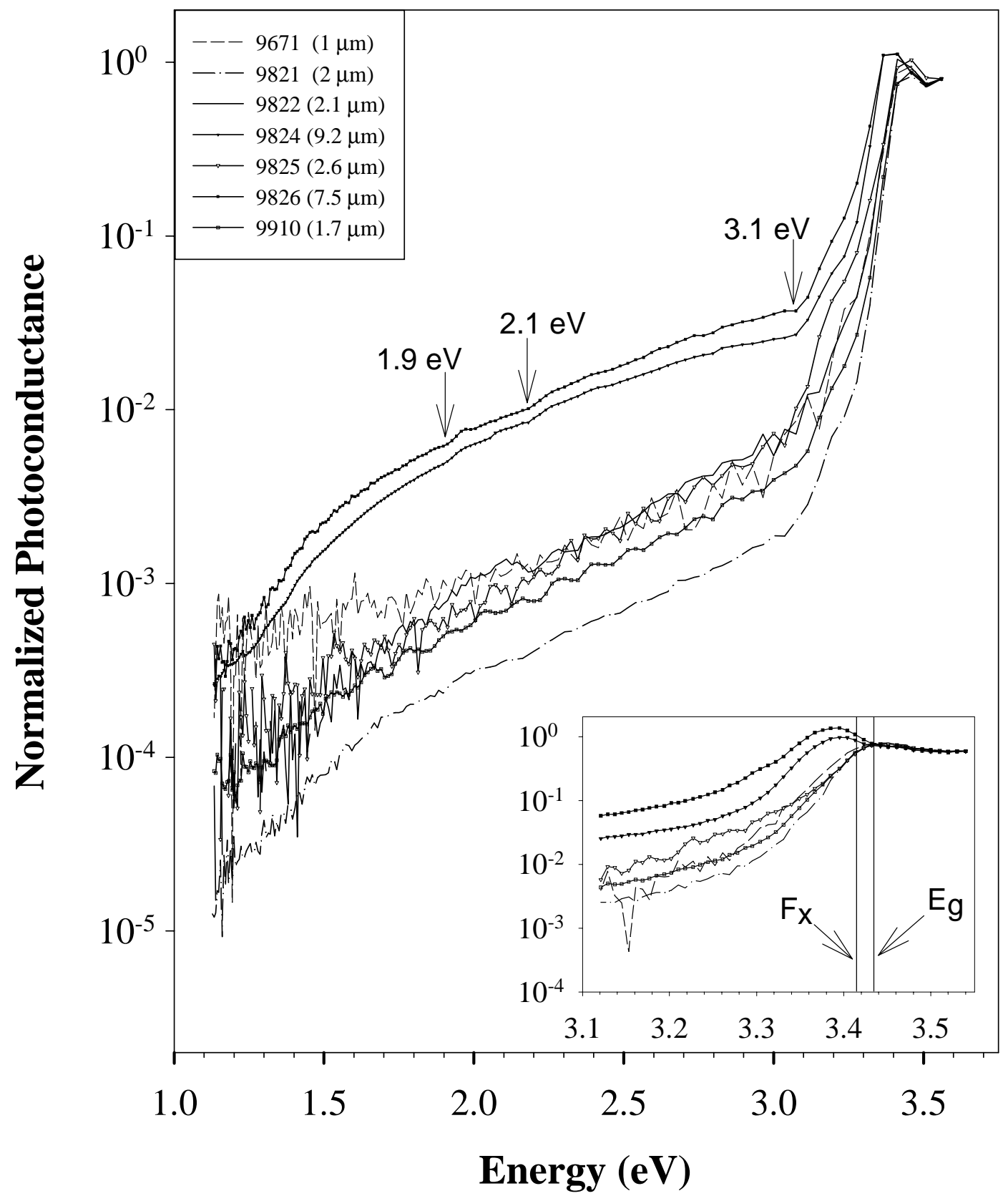

Figure 4.11 Spectral photoconductivity responses for selected GaN samples grown by rf-plasma MBE in our lab. The sample thickness is shown in parentheses. The inset is a close-up of the near band gap region. The $F_{x}$ is the free exciton energy and $E_{g}$ is the band gap energy. 
Initially, we were trying to interpret some PC onset features that can be distinguished in case of samples 9826 and 9824. However, using the second, improved power calibration and repeating the measurements on sample 9824 we did not observe these particular features. A comparison between two separate measurements of the same sample (9824) that use the power calibration curves appropriate to the particular set-up but with the first using the original power meter and calibration and the second the newer power meter and calibration is represented in Figure 4.12. It can be seen that features present in the earlier experiment at $1.92 \mathrm{eV}$ and $2.15 \mathrm{eV}$ in the below band gap region are removed in the later experiment and were apparently experimental artifacts. In this study, we cannot distinguish any definite features in SPC spectra below the band edge that could be related with exact energetic levels of impurities or defects. However, we still can get important information about the defect states by looking at the shape of the photoresponse spectra in the sub-band gap region. It can be see that the photoresponse starts to build up with an onset somewhere below $1.2 \mathrm{eV}$. We cannot say where because of our set-up wavelength limits. The increase continues monotonically until another sharp transition starts at about $3.09 \mathrm{eV}$, which corresponds to near band edge transitions. This type of photoresponse in n-type conducting materials can be interpreted using the model for the defect/impurity density of states distribution in the semiconductor band gap suggested by Qiu et al. ${ }^{32}$ that is illustrated in Figure 4.13. The existence of two band tails is proposed, one that is abrupt and close to the band edges and a gradual tail deeper into the gap. The abrupt tail revealed by the sharply stepped photoresponse near the band edge may reflect the usual Urbach edge, while the deep gradual tail is tentatively attributed to defect states.

Another important feature of the photoresponse spectra represented in Figure 4.11 is the apparent resonance peak centered at $3.39 \mathrm{eV}$, observed for the case of the thicker samples 9824 and 9826 . Since the free exciton binding energy in $\mathrm{GaN}$ is $26-28 \mathrm{meV}^{35}$, which is about the same as the thermal activation energy at room temperature $\left(\mathrm{k}_{\mathrm{B}} \mathrm{T} \approx 25.5 \mathrm{meV}\right)$, then excitonic contribution is expected in absorption-emission processes at room temperature. The reflectance and photoluminescence experimental data confirm its presence at room temperature as shown in Figure 4.14 (a) and (b) with a transition energy of about $3.415 \mathrm{eV}$. Based on these experimental data and on a room temperature study of excitons in photoconductivity spectra, ${ }^{36}$ it was expected that the exciton would contribute to the SPC signal. 


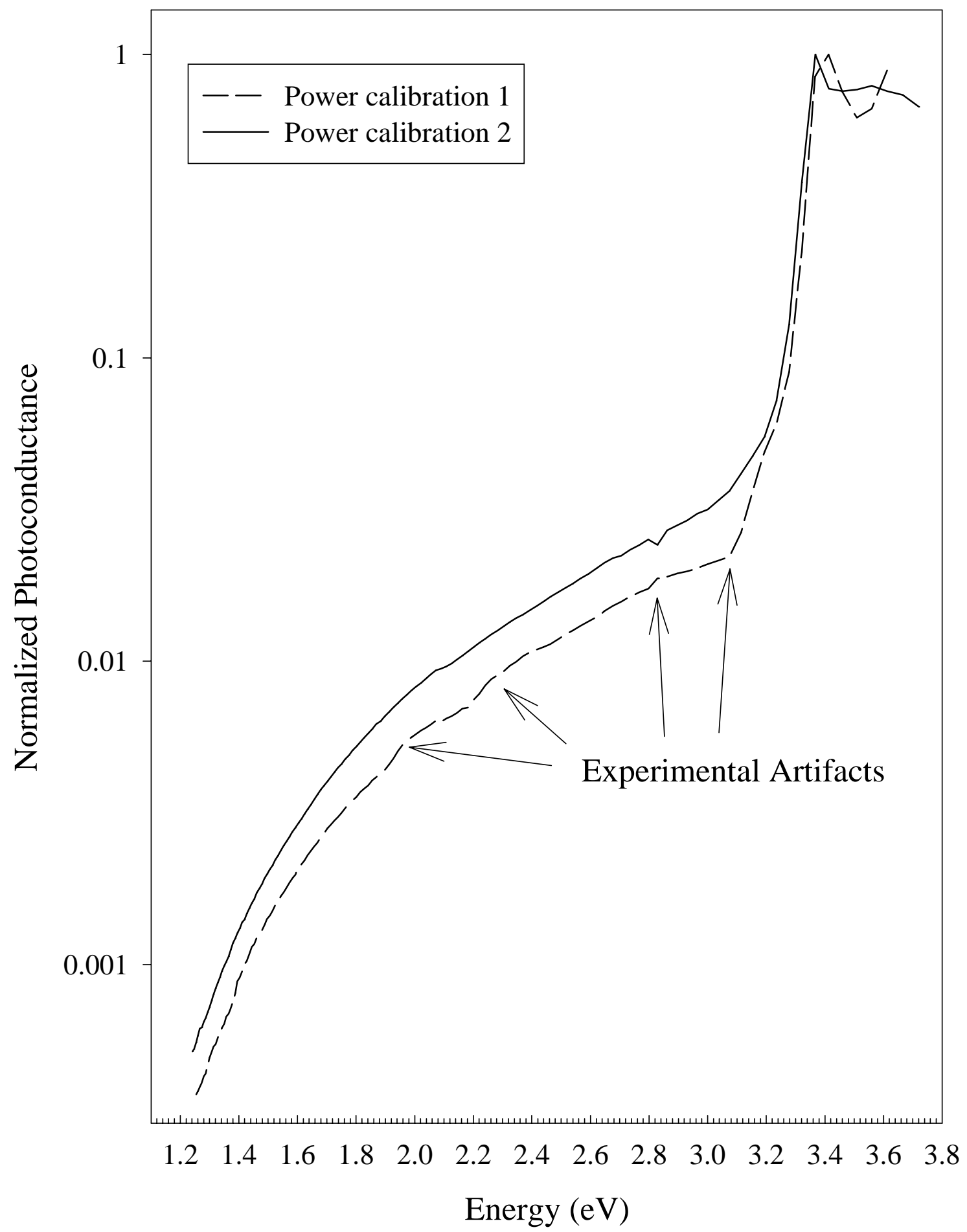

Figure 4.12 Effect of the power calibration on the spectral photoresponse curve of sample 9824 . 
$\log$ (States Available for

Optical Transitions)



Figure 4.13 Schematic distribution of states available for optical transitions in n-type GaN. The states between $E_{c}$ and $E_{v}$ are assumed to be localized states. The arrow represents schematically an optical transition for sub-band gap light absorption. 


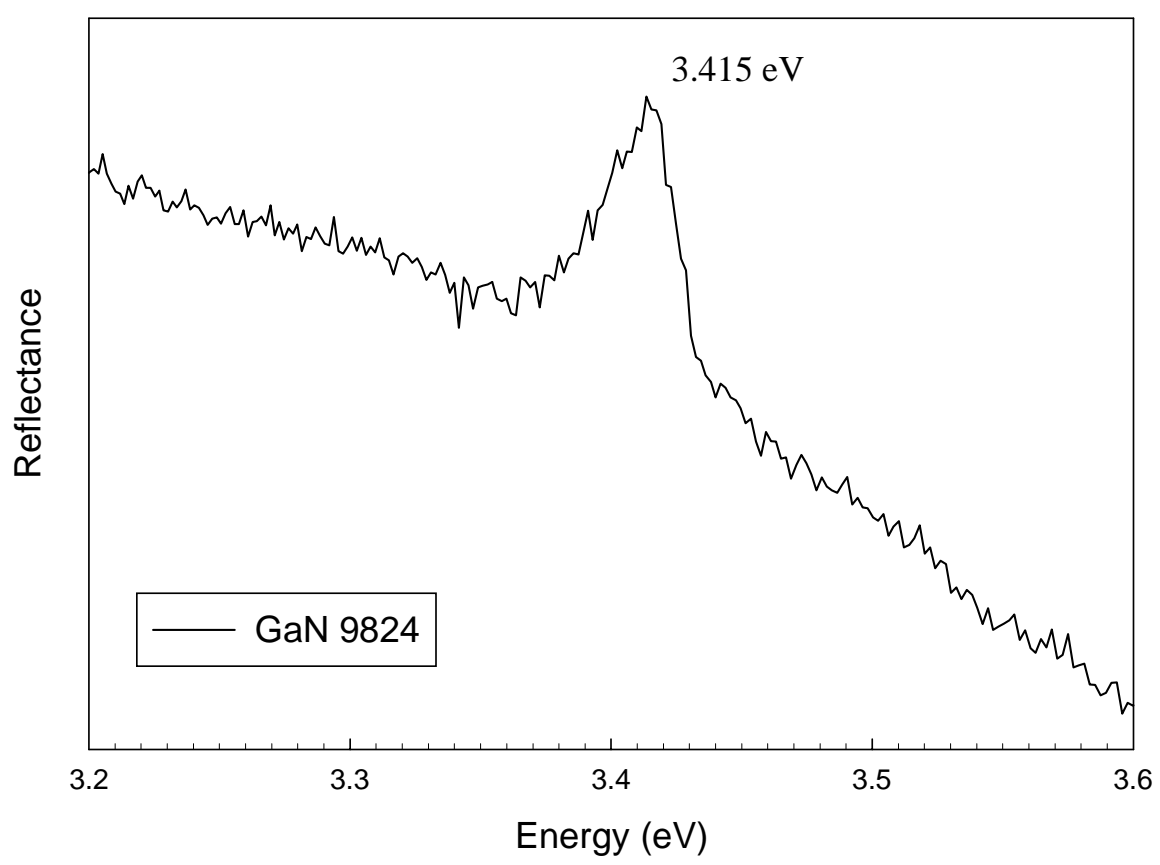

(a)

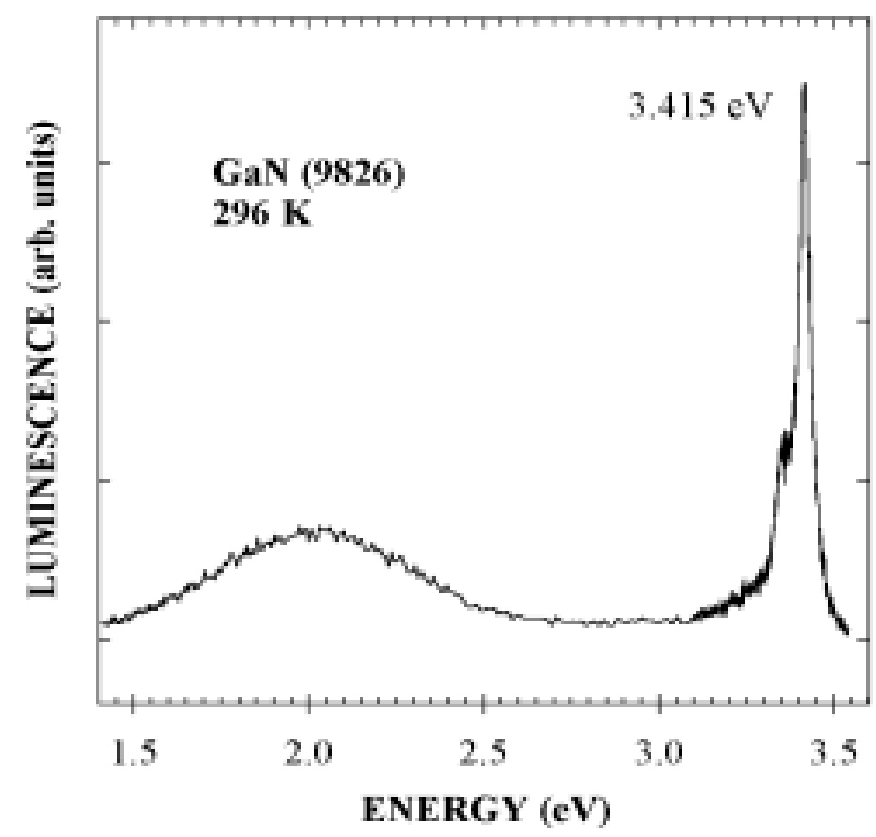

(b)

Figure 4.14 Room temperature reflectance (a) and photoluminescence (b) showing excitonic transitions on MBE GaN samples (9824 and 9826, respectively) 
However, the $3.39 \mathrm{eV}$ peak from our SPC spectra is too low in energy to be an excitonic transition and is thus suspected to be related to an unidentified "impurity" rather than to an excitonic transition. The other thinner samples do not exhibit the feature at $3.39 \mathrm{eV}$ and their photoresponse signal saturates at the value of the excitonic transition.

Another SPC study was done to observe the effect of $\mathrm{Mg}$ doping of $\mathrm{GaN}$ on the photoresponse spectra. This time the MBE epilayer was grown on top of GaN samples that were previously grown by metal-organic chemical vapor deposition (MOCVD) and were furnished by Xerox-PARC. The MOCVD layers grown on sapphire substrates were n-type conducting and were unintentionally doped during the growth. Sample 9975 is a MBE-grown undoped layer on the MOCVD (B1410) substrate and the SPC experiment was done before and after the MBE growth process. Results are represented in Figure 4.15, showing that the initial peak present at $3.418 \mathrm{eV}$ in the SPC spectrum of the B1410 sample is shifted to $3.409 \mathrm{eV}$ by the growth of the MBE layer on top. A similar sample, but this time Mg doped GaN (9979), was also grown on MOCVD GaN (B1647). The SPC signal response is somewhat similar to the previous case: the saturation PC point of $3.408 \mathrm{eV}$ for the B1647 sample is shifted to the lower energy of $3.394 \mathrm{eV}$ by the contribution of the $\mathrm{Mg}$ doped $\mathrm{GaN}$ top layer (Figure 4.16). This is the same energy for the transition revealed in samples 9824 and 9826, suggesting that we have introduced the same type of impurity when growing sample 9979 as in the case of growing samples 9824 and 9826. Interestingly, the intensity of the resonance peak for the $\mathrm{Mg}$ doped sample is the most intense from those measured in our lab, suggesting that the impurity contribution is stronger.

Finally, two hydride vapor phase epitaxy (HVPE) samples were measured in the SPC setup. Comparison of the two samples, LH 1089 and LH 735 featuring the near band edge peak and MBE grown 9910 and 9824, one without and the other with peak. It is observed that one HVPE sample (LH 1089) has the PC saturation point at the band gap energy but the other saturates at the same energy corresponding to the excitonic transition as our sample 9910. In addition, sample LH 735 starts to build up a feature in its shape at about the same energy as the peak of sample 9824.

At this point, the interpretation of the near band edge transitions is not very conclusive, but it can be noted that specific transitions that correspond to different growth conditions can be measured providing particular energy values. The set of samples listed in Table 4.3 exhibit either a resonance peak in SPC at $3.39 \mathrm{eV}$ or a SPC saturation point corresponding to the excitonic 


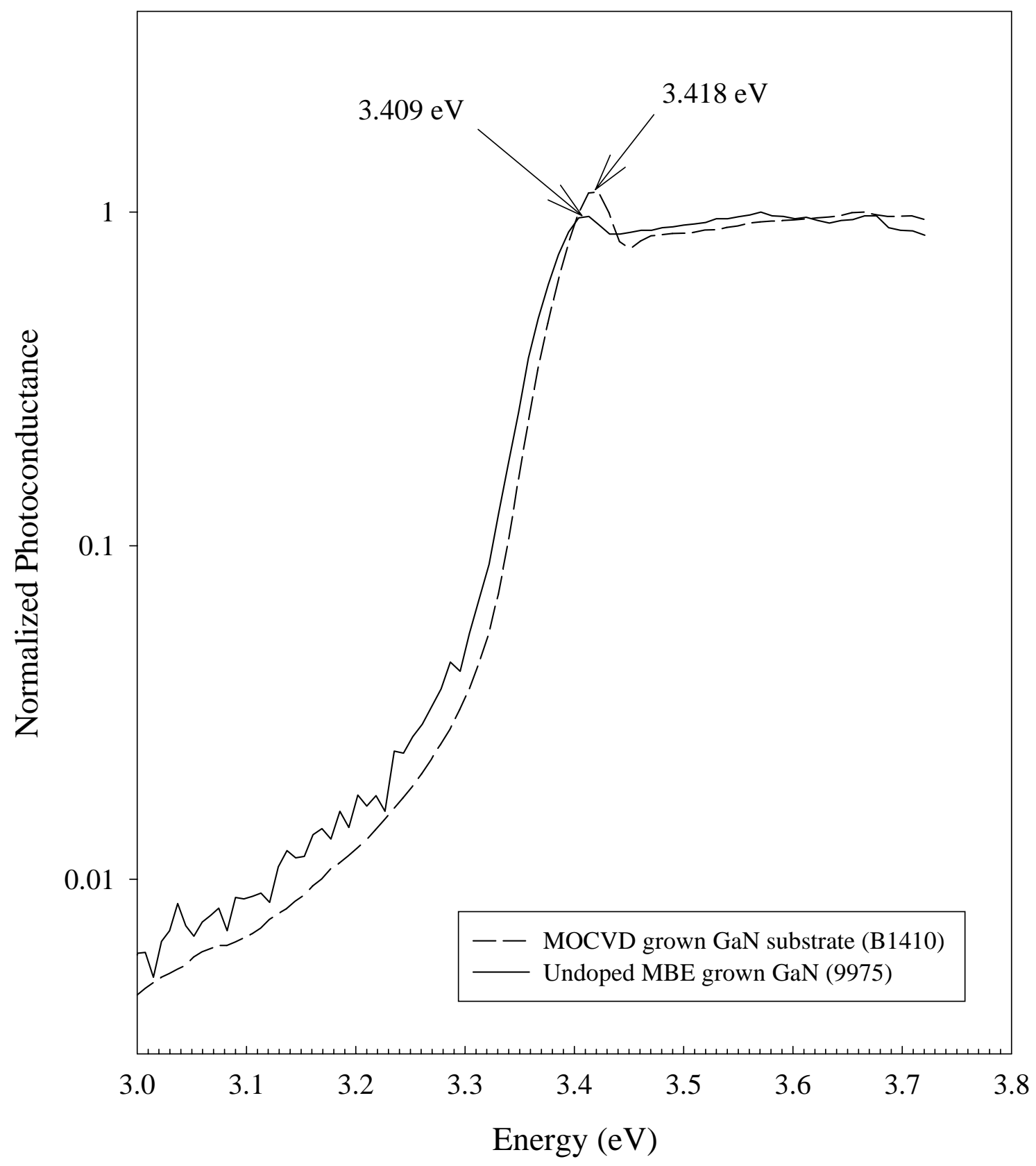

Figure 4.15 SPC response comparison of the MOCVD grown GaN and the MBE grown GaN on top of it. 


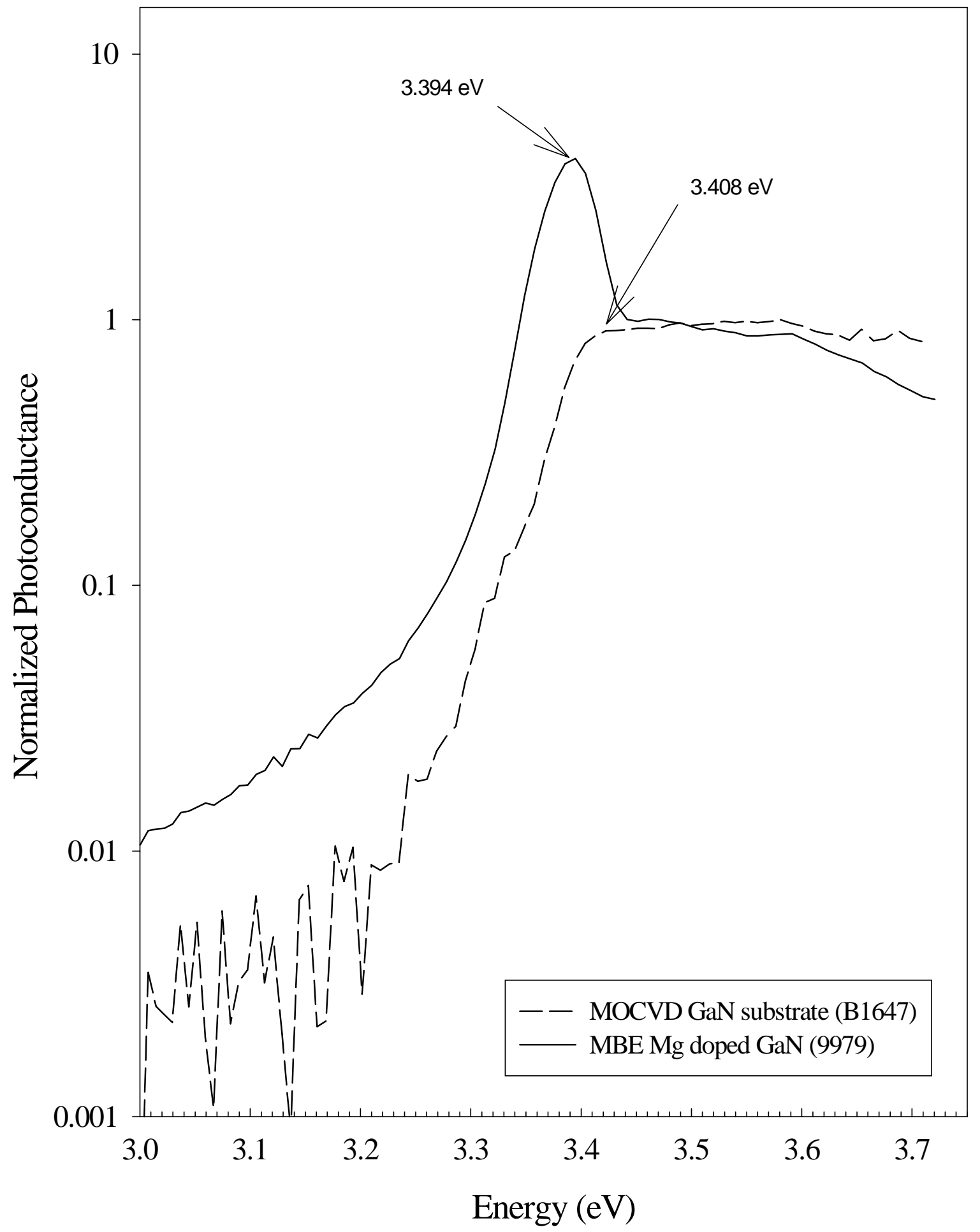

Figure 4.16 SPC response comparison of the MOCVD grown GaN and the Mg doped MBE grown $\mathrm{GaN}$ on top of it. 


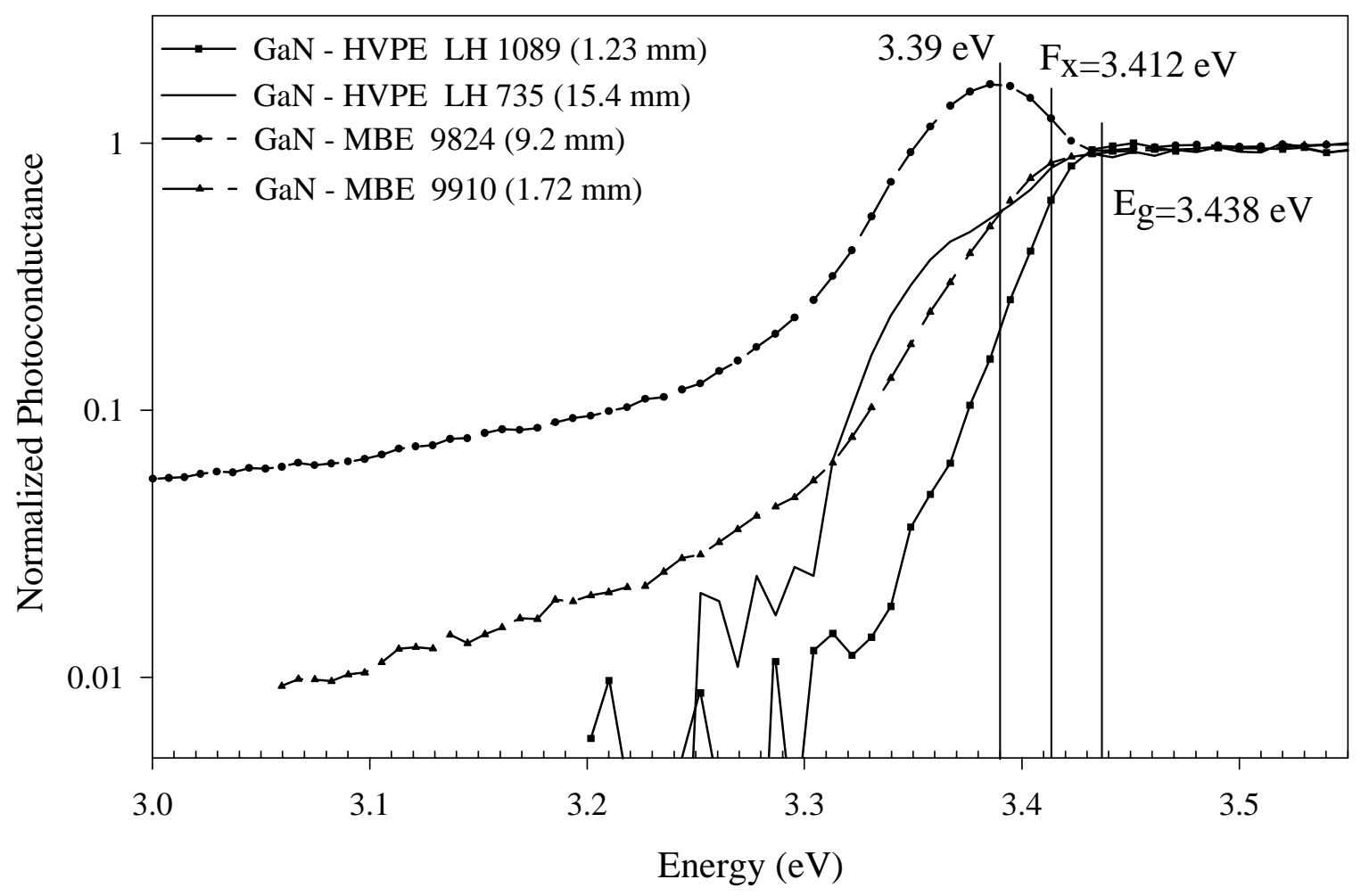

Figure 4.17 Photoconductivity spectra comparison for HVPE and MBE grown GaN. The thickness of each sample can be found in parentheses. 
transition energy. In the case of the MBE samples grown on MOCVD GaN substrates, the initial MOCVD sample PC saturation point or the resonance peak that is close to the excitonic energy is shifted to lower energies toward the $3.39 \mathrm{eV}$ level by the contribution of the MBE layer. This type of phenomenon could be explained by assuming that MBE growth systematically introduces some unwanted "impurity" seen in SPC spectra. The same type of impurity may be present in the LH 735 HVPE sample that starts to build up a feature at an energy between 3.3 and $3.4 \mathrm{eV}$. Future complementary photoluminescence studies may help to explain the "peak" effects that were observed in this work.

The use of the constant current source to supply the voltage to the sample studied by SPC helped to improve the signal to noise ratio in some of the samples. Two samples measured initially with the battery as a voltage source that had noisy responses in the sub-band energy region were measured again with the constant current source, this time exhibiting nice interference effects with the light reflected from the sapphire substrate. The results are shown in Figure 4.18. Analysis of the interference patterns using refraction indices from literature determined the sample thickness. For 9821 a thickness of $1.87 \mu \mathrm{m}$ and for 9910 a thickness of $1.92 \mu \mathrm{m}$ were obtained. These values are in agreement with the values obtained by transmittance measurements that are used to find the sample thickness and can be found in Table 4.3. 


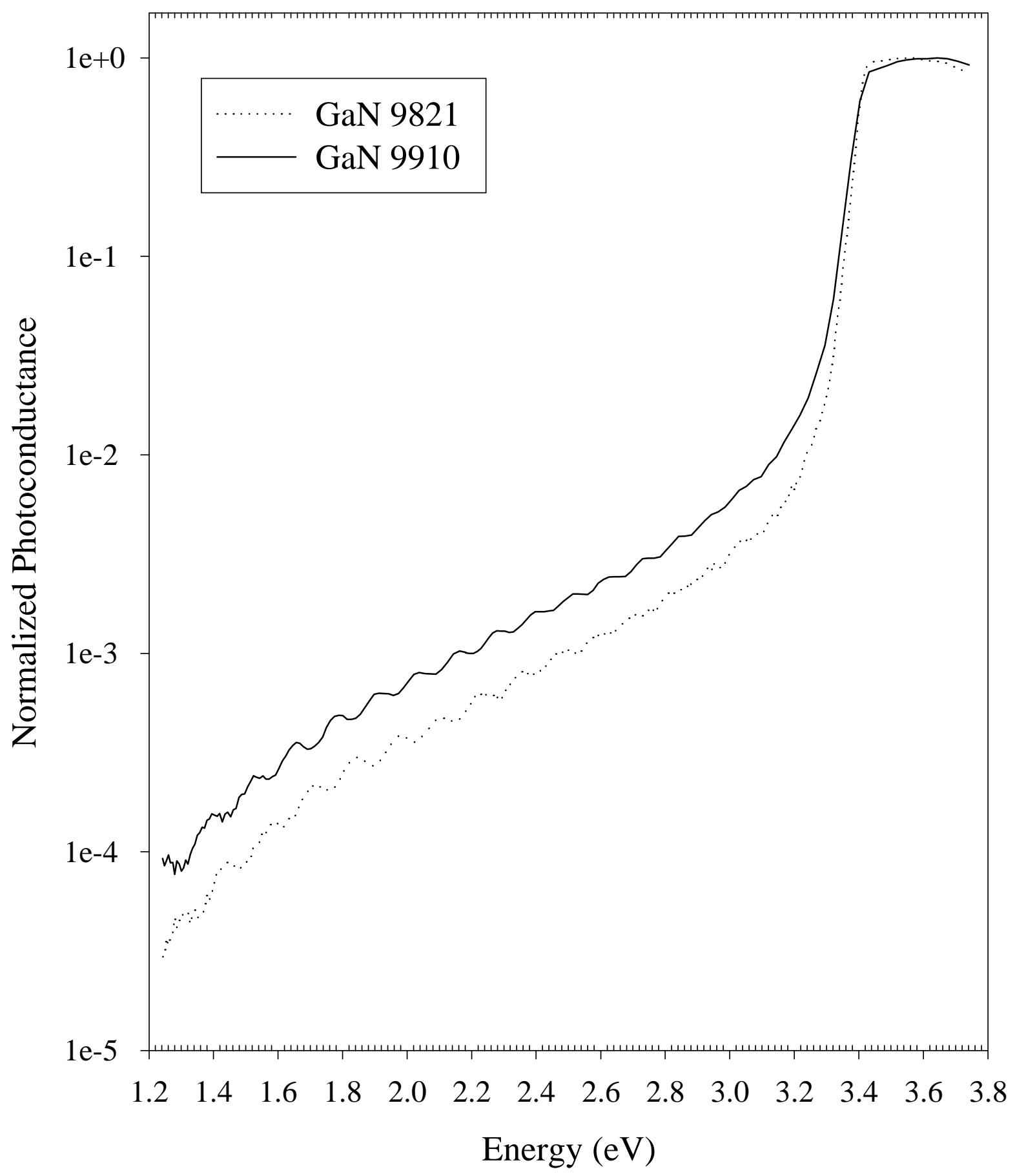

Figure 4.18 Interference effects in the sub-band gap region used to calculate the sample thickness. 


\section{Summary and suggested future research}

Progress in compound semiconductor growth technologies is made continuously with the demand to produce high quality materials, control their composition and move forward to produce applications. Two types of compound semiconductor materials were considered in this study: the ternary bulk $\mathrm{Cd}_{1-\mathrm{x}} \mathrm{Zn}_{\mathrm{x}}$ Te system and the epitaxial GaN system. In the case of $\mathrm{Cd} 1$. ${ }_{\mathrm{x}} \mathrm{Zn}_{\mathrm{x}} \mathrm{Te}$, material it is important to achieve fine control over its composition to provide latticematched substrates for the growth of $\mathrm{Hg}_{1-\mathrm{x}} \mathrm{Cd}_{\mathrm{x}} \mathrm{Te}$, which is important for infrared detection. In the other case, the lack of lattice matched substrates for the growth of epitaxial GaN still provides material with considerable defect densities despite the substantial amount of research on this material date. The optical characterization techniques that were used in the present work represent powerful tools for investigating material issues. Photoreflectance spectroscopy can make accurate composition determinations of $\mathrm{Cd}_{1-\mathrm{x}} \mathrm{Zn}_{\mathrm{x}} \mathrm{Te}$ via energy level determination, and photoconductivity spectroscopy can be used to study defects and imperfections in the band gap of GaN. To build the experimental set-ups for these two techniques and apply them to the materials of interest were the principal objectives that motivated this research. The results obtained demonstrate that the objectives were successfully accomplished.

Optimization of the experiment set-ups conditions to provide maximum responses in the PR and PC spectra was accomplished by considering each component of the experimental arrangement and its influence on the spectra. The aim of minimizing instrumental perturbations to the measured spectra was attained by making successive measurements and varying conditions for one set-up component at a time. In this way, it was assured that the observed spectra were providing only physical information about the sample under study and not experimental uncertainties.

After refinement the PR set-up allowed the measurement of high resolution, repeatable spectra. The band gap energy was accurately determined considering either excitonic or excitonic and band gap contributions to the photoreflectance spectra. Consecutively, the composition of $\mathrm{Cd}_{1-\mathrm{x}} \mathrm{Zn}_{\mathrm{x}}$ Te compounds was determined using the band gap energy values. It is concluded that the main uncertainty of the band gap determination is the fitting procedure when using a double transition contribution, which has a large number of fitting parameters that are not easily controlled. Further studies can optimize the PR spectra for obtaining only excitonic 
contributions that are easier to control in the fitting procedure and obtain accurate band gap energy data more easily. This might be possible through the use of an adequate laser power for each sample as some preliminary results have revealed. To understand the effect of the laser on the built-in surface electric field and make quantitative evaluations about its strength and/or interaction with the laser incident radiation, future studies may focus on the Franz-Keldysh oscillations. ${ }^{24}$ Complementary optical characterization techniques such as photoluminescence at room temperature may confirm the assumption that in the case of the samples considered the excitonic contribution is the dominant one.

Spectral photoconductivity was successfully used to study transitions near the band edge of epitaxial layers of GaN, obtained by various growth methods. It is observed that in the case of photoconductivity studies, further progress can be made by using a real time monitoring of the intensity of the incident light on the sample, eliminating the uncertainties related to the influence of the power calibration. Another set-up change can be attempted to surpass the difficulty of measuring samples with small signal to noise ratio by using a sample electrical circuit similar to the one described in Reference 26, page 48. To make quantitative comparisons between various measured samples, future experiments need to take into account the sample thickness to normalize the photoconductance spectra. 


\section{References}

1. J.-Y. Duboz, Phys. Stat. Sol. (a) 176 (5), 5 (1999).

2. H. Morkoc, S. Strite, G.B.Gao, M.E. Lin, B. Sverdlov, and M. Burns, J. Appl. Phys. 76, 1363 (1994).

3. S. Yoshida and J. Suzuki, Jpn. J. Appl. Phys. 37, L482 (1998).

4. Compound Semiconductors 5 (4), 16 (1999).

5. Compound Semiconductors 5 (2), 12 (1999).

6. O. Ambacher, J. Phys. D: Appl. Phys. 31, 2653 (1998).

7. S. Nakamura, M. Senoh, S.I. Nagahama, T. Matsushita, K. Kiyoku, Y. Sugimoto, T. Kozaki, H. Umemoto, M. Sano, and T. Mukai, Jpn. J. Appl. Phys. 38 (3A), L226 (1999).

8. S. Strite and H.Morkoc, J. Vac. Sci. Technol. B 10 (4), 1237 (1992).

9. G.M. Smith, J.M Redwing, R.P. Vaudo, E.M. Ross, J.S. Flynn, and V.M. Phanse, Appl. Phys. Lett., 75 (1), 25 (1999).

10. J.C. Carrano, T. Li, D.L. Brown, P.A. Grudowski, C.J. Eiting, R.D. Dupuis, and J.C. Campbell, Elec. Lett., 34 (18), 1779 (1998).

11. L. Verger, J.P Bonnefoy, F. Glasser and P. Ouvrier-Buffet Leti, J. Electron. Mat. 26 (6), 738 (1997).

12. C. Scheiber, Nucl. Inst. and Meth A 380, 385 (1996).

13. Y. Eisen and A. Shor, J. Cryst. Growth 184-185, 1302 (1998).

14. Y. Eisen, A. Shor and I. Mardor, Nucl. Inst. and Meth A 428, 158 (1999).

15. F.G. Sanchez-Almazan, H. Navarro-Contreras, G. Ramirez-Flores, M.A. Vidal, O. Zelaya-Angel, M.E. Rodriguez, and R. Baquero, J. Appl. Phys. 79 (10), 7713 (1996).

16. R.H. Bube, Photoconductivity of Solids, Wiley, New York, (1960).

17. O.J. Glembocki and B.V. Shanabrook, in Semiconductors and Semimetals 36, chapter 4, 239 (1987), ed. by David G. Seiler, and Christopher L. Littler

18. M. Cardona, Modulation Spectroscopy, Academic Press, New York, (1969).

19. B.O. Seraphin and N. Bottka, Phys. Rev. 145, 628 (1966).

20. F.H. Pollak, in Group III Semiconductor Compounds, Clarendon Press, Oxford, Chapter 5, (1998), ed. By Bernard Gil. 
21. F.H. Pollak and H. Shen, J. of Electron. Mat. 19 (5), 399 (1990).

22. F.H. Pollak, Proceedings of the SPIE 276, 142 (1981).

23. O.J. Glembocki, B.V. Shanabrook, N. Bottka, W.T. Beard, and J. Comas, Appl. Phys. Lett. 46, 970 (1985).

24. H. Shen and M. Dutta, J. Appl. Phys. 78 (4), 2151 (1995).

25. D.E. Aspnes, Surface Sci. 37, 418 (1973).

26. N.V. Joshi, Photoconductivity: art, science and technology, New York: Marcel Dekker, (1990).

27. R.H. Bube, Electronic Proprieties of Crystalline Solids, Academic Press, New York, (1974).

28. F.H. Pollack and O.J. Glembocki, Proceedings of the SPIE 946, 2 (1988).

29. H. Shen and M. Dutta, Appl. Phys. Lett. 57, 587 (1990).

30. S.P. Tobin, J.P. Tower, P.W. Norton, D. Chandler-Horowitz, P.M. Amirtharaj, V.C. Lopes, W.M. Duncan, A.J. Syllaios, C.K. Ard, N.C. Giles, J. Lee, R. Balasubramanian, A.B. Bollong, T.W. Steiner, M.L.W. Thewalt, D.K. Bowen, and B.K. Tanner, J. Electron. Mat. 24 (5), 697 (1995).

31. Z. Yu, S.G. Hofer, N.C. Giles, and T.H. Myers, Phys. Rev. B 51 (19), 51 (1995).

32. C.H. Qiu, C. Hoggatt, W. Melton, M.W. Leksono and J.I. Pankove, Appl. Phys. Lett. 66 (20), 2712 (1995).

33. S.C. Jain, M. Willander, J. Narayan, R. Van Overstraeten, J. of Appl. Phys. 87 (3), 965 (2000).

34. A.J. Ptak, V.A. Stoica, L.J. Holbert, M. Moldovan, and T.H. Myers, MRS Internet J. Nitride Semicond. Res. 5S1, W11.45 (2000).

35. A.K. Viswanath, J.I. Lee, S. Yu, D. Kim, Y. Choi and C. Hong, J. Appl. Phys. 84, 3848 (1998).

36. F. Binet, J.Y. Duboz, E. Rosencher, F. Scholz, and V. Harle, Phys. Rev. B 54 (11), 8116 (1996). 